\title{
A Neural Basis for Developmental Topographic Disorientation
}

\author{
Jiye G. Kim, ${ }^{1 \star}$ Elissa M. Aminoff, ${ }^{3,4 \star}$ Sabine Kastner, ${ }^{1,2}$ and ${ }^{\circledR}$ Marlene Behrmann ${ }^{3,4}$ \\ ${ }^{1}$ Princeton Neuroscience Institute and ${ }^{2}$ Department of Psychology, Princeton University, Princeton, New Jersey 08644, and ${ }^{3}$ Center for the Neural Basis of \\ Cognition and ${ }^{4}$ Department of Psychology, Carnegie Mellon University, Pittsburgh, Pennsylvania 15213
}

Developmental topographic disorientation (DTD) is a life-long condition in which affected individuals are severely impaired in navigating around their environment. Individuals with DTD have no apparent structural brain damage on conventional imaging and the neural mechanisms underlying DTD are currently unknown. Using functional and diffusion tensor imaging, we present a comprehensive neuroimaging study of an individual, J.N., with well defined DTD. J.N. has intact scene-selective responses in the parahippocampal place area (PPA), transverse occipital sulcus, and retrosplenial cortex (RSC), key regions associated with scene perception and navigation. However, detailed fMRI studies probing selective tuning properties of these regions, as well as functional connectivity, suggest that J.N.'s RSC has an atypical response profile and an atypical functional coupling to PPA compared with human controls. This deviant functional profile of RSC is not due to compromised structural connectivity. This comprehensive examination suggests that the RSC may play a key role in navigation-related processing and that an alteration of the RSC's functional properties may serve as the neural basis for DTD.

Key words: developmental topographic disorientation; navigation; parahippocampal place area; retrosplenial cortex; scene perception; transverse occipital sulcus

\section{Significance Statement}

Individuals with developmental topographic disorientation (DTD) have a life-long impairment in spatial navigation in the absence of brain damage, neurological conditions, or basic perceptual or memory deficits. Although progress has been made in identifying brain regions that subserve normal navigation, the neural basis of DTD is unknown. Using functional and structural neuroimaging and detailed statistical analyses, we investigated the brain regions typically involved in navigation and scene processing in a representative DTD individual, J.N. Although scene-selective regions were identified, closer scrutiny indicated that these areas, specifically the retrosplenial cortex (RSC), were functionally disrupted in J.N. This comprehensive examination of a representative DTD individual provides insight into the neural basis of DTD and the role of the RSC in navigation-related processing.

\section{Introduction}

The ability to navigate around one's environment is a complex skill that is critical for everyday life. Damage to multiple brain regions or "nodes" that are implicated in this navigational ability

\footnotetext{
Received Feb. 13, 2015; revised Aug. 14, 2015; accepted Aug. 18, 2015.

Author contributions: J.G.K., E.M.A., S.K., and M.B. designed research; J.G.K., E.M.A., and M.B. performed research; J.G.K. and E.M.A. analyzed data; J.G.K., E.M.A., S.K., and M.B. wrote the paper.

This work was supported by National Institutes of Health (Grant MH64043 to S.K. and Grant EY02316601 to J.G.K.), the National Science Foundation (Grants BCS0923763 and SBE0542013 to M.B., Grant BCS1328270 to S.K.), and the Office of Naval Research (MURI Grant N000141010934 to E.M.A.). We thank J.N. for participating in the study, Ford Burles for providing us with J.N.'s scores from www.gettinglost.ca, and Ryan Egan for assisting with the data collection.

The authors declare no competing financial interests.

*J.G.K. and E.M.A. are co-first authors

Correspondence should be addressed to Jiye G. Kim, Princeton Neuroscience Institute, Princeton University, Washington Road, Princeton, NJ 08644. E-mail: jiyekim@princeton.edu.

DOI:10.1523/JNEUROSCI.0640-15.2015

Copyright $\odot 2015$ the authors $\quad 0270-6474 / 15 / 3512954-16 \$ 15.00 / 0$
}

results in impairment. For example, patients with damage to the posterior parietal cortex are impaired at representing locations of objects with respect to themselves (Stark, 1996; Aguirre and D'Esposito, 1999). Patients with damage to the occipitotemporal (Whiteley and Warrington, 1978; Aguirre et al., 1998) and/or the parahippocampal (Epstein et al., 2001; Mendez and Cherrier, 2003) regions have difficulties navigating due to poor recognition of previously familiar landmarks. Patients with damage to the retrosplenial cortex (RSC), although able to recognize familiar landmarks, are unable to encode and derive directional information from landmarks (e.g., the direction of a familiar location in relation to another familiar location; Takahashi et al., 1997; Aguirre and D'Esposito, 1999; Katayama et al., 1999; Maguire, 2001; Hashimoto et al., 2010). Finally, patients with damage to medial temporal lobe structures (Habib and Sirigu, 1987) show topographic disorientation due to the inability to represent newly learned routes and environments. 
Impairments in spatial navigation, however, may manifest even without explicit brain damage. Individuals with developmental topographic disorientation (DTD) suffer from severe, life-long navigational deficits in the absence of an obvious neurological condition or structural damage (Iaria et al., 2009, 2014; Bianchini et al., 2010, 2014; Palermo et al., 2014). These individuals have problems representing a spatial layout, repeatedly getting lost in familiar environments, and having difficulties learning new routes. Since the first report in 2009, >120 cases have been documented (Iaria and Barton, 2010). Although DTD individuals may vary in the nature and severity of their navigational abilities, they have in common substantial difficulty forming a cognitive map or mental representation of their environment, even of a familiar scene, while retaining the ability to recognize landmarks such as distinctive buildings. Although the neural mechanism underlying DTD is not known, the behavioral profile most closely resembles the behavioral deficits of patients with retrosplenial damage, as described above. Given that there is no overt structural damage in DTD, there may be functional deficits within the RSC or the RSC's interaction with other brain regions involved in different aspects of navigational processes.

Here, we report a comprehensive neuroimaging study using fMRI and diffusion tensor imaging (DTI) to explore the neural basis of DTD in a single patient, J.N. Although it is still unclear whether DTD is a heterogeneous condition in which the inability to form and use a cognitive map is one aspect of a multiplicity of symptoms or if it is a more homogenous condition related to a deficit in constructing a cognitive map, J.N.'s profile strongly resembles the model DTD pattern, so the results from this well characterized representative case may be applicable to DTD more generally.

Previous fMRI studies in healthy individuals demonstrate that several brain regions, such as the parahippocampal place area (PPA), transverse occipital sulcus (TOS), and RSC, are activated in visual scene and navigational processing (Epstein and Kanwisher, 1998; Epstein et al., 2007b). These regions show selectively greater responses to scenes than to other categories of stimuli and are considered critical for processing visual information in the service of navigation (Epstein et al., 2001; Epstein, 2008). Because the behavioral profile of DTD involves navigational and spatial processes, we examined all three regions related to scene and navigational processes. Our experiments compared the functional properties of these regions in J.N. and controls using functional localizers and fMRI adaptation experiments. We also conducted analyses of functional and structural connectivity between these areas. The key findings indicate that the functional, but not the structural, profile of J.N.'s scene network, especially of the RSC, was disrupted. This study provides the most extensive neuroimaging investigation of the neural mechanisms underlying DTD to date and also offers insights into the neural basis of navigability in normal vision.

\section{Materials and Methods}

Participants

J.N., a 56 year-old, left-handed (score of -50 on Edinburgh Handedness Inventory; Oldfield, 1971) woman self-employed as a speech and language pathologist, and 13 control subjects (all female, age range 24-60, mean: 41.6 years, 3 left-handed) participated in the MRI experiments performed at Carnegie Mellon University (CMU) Scientific Imaging and Brain Research Center (for J.N. and control participant C1) and Princeton University (PU; for 12 controls). Six control participants were age matched to J.N. and three were handedness matched to J.N. For all analyses, the six age-matched controls did not differ from the other non- aged-matched participants, so the control data were collapsed across all participants (see Results section for a comparison between two age groups). An additional six female age-matched controls (age range: 5260, mean: 55.7) were recruited to perform the behavioral experiments at PU. All participants had normal or corrected-to-normal vision and no history of neurological disorder. All participants gave informed consent and those who participated in the imaging studies were screened for safety in accordance with the procedures approved by CMU's and PU's Institutional Review Boards.

The statistical comparison between J.N. and the control group was done with a modified independent samples two-tailed $t$ test (Crawford and Howell, 1998), which tests the null hypothesis that the results of the single case is drawn from the population of controls. This method has been widely used in neuropsychological studies (Behrmann et al., 2006; Konen et al., 2011) and has advantages over other single case statistics such as the modified ANOVA or $z$-score inferences (Crawford et al., 2004).

As a visual benchmark to reference J.N.'s results, the results of $\mathrm{C} 1$ (who is age, handedness, and scanner matched to J.N.) are displayed in the figures as well. To ensure that $\mathrm{C} 1$ was a representative control, we compared Cl's data with the data from the other controls $(n=12)$ and found no difference between $\mathrm{C} 1$ and the rest of the controls' data (see Results).

\section{Case history: general description}

J.N. has a master's degree and lives a full and active life with her husband, who referred her to us. J.N.'s husband offered an anecdotal description of her abilities, stating that,

"She has severe visual-spatial deficits, and in particular [she] cannot make a 'mental map'. She'll not recognize a street she's driven on many, many times. She does not have a facial recognition deficit, but visualizing places in space is impossible. When at the movie theater, if she goes to the bathroom, she does not know how to get back to her seat. Or, if she leaves a building and then tries to reenter it, she will not remember where the entrance is and has to walk around the periphery until she finds the entrance. She gets lost easily, and often and for years, [she] was accused of not paying attention. On the other hand, she's extremely smart verbally; she reads far faster than me."

J.N. has a brother who has been diagnosed as high functioning on the autism spectrum disorder, but J.N. herself was not. Aside from a kidney transplant in 2001, her medical history is unremarkable.

Quantitative analyses detailing J.N.'s specific behavioral deficits can be found in the "Behavioral results" section. In brief, J.N.'s performance on navigation-related processes such as mental rotation or spatial manipulation is significantly poorer than the controls and mirrors that of previously reported cases of DTDs. In contrast, her performance on basic perceptual abilities such as face, object, and scene recognition is indistinguishable from that of the controls and her results on tests of nonspatial memory processing such as word recall, digit span, and face identity memory test are all within the normal range. These results confirm that J.N.'s navigational deficits are not due to a general perceptual or memory problem.

\section{Experimental procedure}

J.N. and six age-matched controls were tested on a series of behavioral assessments designed to characterize the nature and extent of J.N.'s navigational impairment. These include tests of navigational abilities; face, object, and scene perception; memory; and mental rotation or spatial manipulation.

J.N. and 13 additional participants were scanned in multiple MRI sessions. Scans included retinotopic mapping of early visual areas, functional localizer scans to localize category-selective higher-order visual areas, two fMRI adaptation experiments, resting-state scans, anatomical scans, and a DTI scan.

\section{Neuroimaging methods}

MRI acquisition and preprocessing. Scanning was performed with a Siemens Verio 3T scanner at CMU (for J.N. and C1) and a Siemens Skyra 3T scanner at PU. The imaging parameters for all MRI scans were identical across the two scanning facilities. High-resolution T1-weighted anatomical scans were obtained using the MPRAGE sequence TR, $2.3 \mathrm{~s}$, TE, 1.97 
$\mathrm{ms}$, flip angle, $9^{\circ}, 256 \times 256$ matrix, $1 \mathrm{~mm}^{3}$ resolution, 179 volumes. The fMRI scans were acquired with a $\mathrm{T} 2{ }^{\star}$-weighted echo planar sequence; for retinotopy and functional localizers: $\mathrm{TR}=2.5 \mathrm{~s}, \mathrm{TE}=30 \mathrm{~ms}$, flip angle $=$ $83^{\circ}, \mathrm{FOV}=192$, voxel resolution $=3 \mathrm{~mm}^{3}$, 39 slices; resting state: $\mathrm{TR}=$ $1.8 \mathrm{~s}, \mathrm{TE}=30 \mathrm{~ms}$, flip angle $=68^{\circ}, \mathrm{FOV}=192$, voxel resolution $=3 \times$ $3 \times 4 \mathrm{~mm}, 32$ slices; adaptation experiments: $\mathrm{TR}=2 \mathrm{~s}, \mathrm{TE}=29 \mathrm{~ms}$, flip angle $=79^{\circ}, \mathrm{FOV}=192$, voxel resolution $=3 \mathrm{~mm}^{3}, 36$ slices. DTIs were acquired with diffusion weights isotropically distributed along 50 directions using a $b$ value of $2400 \mathrm{~s} \times \mathrm{mm}^{2}: \mathrm{TR}=6.1 \mathrm{~s}, \mathrm{TE}=118 \mathrm{~ms}$, voxel resolution $=2.5 \mathrm{~mm}^{3}$, 40 slices.

The functional scans were preprocessed using AFNI (http://afni.nimh. nih.gov/afni), including despiking, slice time correction, motion correction, and detrending. Data were smoothed with a $4 \mathrm{~mm}$ Gaussian kernel FWHM and normalized to percentage signal change by dividing the time series by its mean intensity. The resting-state runs were band-passed filtered with 0.1 and $0.01 \mathrm{~Hz}$. All functional scans were coregistered to each session's structural scan and the structural scans were aligned across sessions. The software packages FreeSurfer (http://surfer.nmr.mgh. harvard.edu) and SUMA (http://afni.nimh.nih.gov/afni/suma/) were used for the cortical thickness analysis and for producing inflated and flat cortical surface reconstructions.

Retinotopic mapping. Retinotopic areas including V1, V2, V3, V4, V3a, V3b, and MT were defined for each subject (see Fig. $1 C$ for retinotopic ROIs in J.N. and C1). A colored checkerboard with the chromaticity and luminance of each square alternating at a flicker frequency of $4 \mathrm{~Hz}$ rotated around a central fixation at a rate of $9 \% \mathrm{~s}$ with a total rotation of 8 cycles per run. The radius of the wedge spanned $1-14^{\circ}$ in eccentricity and had an arc length of $45^{\circ}$. At every $3-5 \mathrm{~s}, 1$ of the rings of the wedge (with a random distance from fixation) dimmed and subjects were instructed to detect the dimming of the ring with a button press while maintaining central fixation. Each run started and ended with a blank screen with a center fixation cross for $10 \mathrm{~s}$ and the duration of a run was $5.7 \mathrm{~min}$. Three retinotopy runs were acquired for each subject and, across different runs, the checkerboard wedge rotated clockwise or counterclockwise. To delineate visual areas, a Fourier analysis was used to determine the amplitude and phase of the harmonic at the stimulus frequency. The statistical threshold used to delineate ROIs was $p \leq 0.001$, uncorrected, derived from the F ratio from the Fourier transform. Similar phase encoding parameters and procedures have been used previously (Bandettini et al., 1993; Swisher et al., 2007), as have the statistical analyses (Schneider et al., 2004; Arcaro et al., 2009). All of the retinotopic areas listed above were defined in all subjects except for one control subject, in whom MT was not localized due to signal dropout in that region.

Functional localizers. Standard block design functional localizer (Kim et al., 2009; Kim and Biederman, 2011) runs were acquired for each subject. Each run (5.3 min) began and ended with a fixation cross for $15 \mathrm{~s}$. Each run contained four repetitions of four blocks alternating between images of colored scenes, faces, objects, and grid-scrambled objects (see Fig. 1A), resulting in a total of 12 blocks for each category condition. The scenes used in the localizer runs differed from those used in the adaptation experiments. For each repetition, the ordering of the condition blocks was randomized. Each block lasted $15 \mathrm{~s}$ and consisted of 20 exemplars randomly selected without replacement from a total of 40 exemplars per stimulus category. Each stimulus subtended $7.4^{\circ}$ and was presented for $350 \mathrm{~ms}$ with an interstimulus interval (ISI) of $750 \mathrm{~ms}$. The four category conditions were blocked without a gap in between. The repetitions of the conditions were separated by $15 \mathrm{~s}$ of fixation. The fixation cross remained present throughout the run and subjects were instructed to maintain fixation while passively viewing the stimuli.

Square-wave functions matching the time course of the design were convolved and used as regressors of interest in a multiple regression model. Additional regressors included motion parameters, linear drifts within runs, and shifts between runs. The weighted contrast of $[3 \times$ scene $>$ face + object + scrambled] was used to define PPA, TOS, and RSC. The weighted contrast of $[3 \times$ face $>$ scene + object + scrambled $]$ was used to define face-selective fusiform face area (FFA). Objectselective lateral occipital complex (LOC) was defined with the contrast of object $>$ scrambled. For all ROIs, a threshold of $p<0.0001$, uncorrected for multiple comparisons, was used.
In addition to these ROIs, the hippocampus (HIP) was anatomically defined for each participant using the automatic subcortical segmentation tool from FreeSurfer (Fischl et al., 2002). Before this step, manual segmentation of gray matter and white matter (WM) boundary was done on each participant's high-resolution structural scan to ensure more accurate segmentation of HIP.

Adaptation experiments. Each participant was scanned in three runs of the familiar/unfamiliar scenes and three runs of the indoor/outdoor scenes. In alternating blocks, participants viewed either a series of different scenes (new) or repetitions of an identical scene (repeat). The different scene types (e.g., familiar or unfamiliar) were assigned randomly to blocks. Each run consisted of a total of 12 blocks (either new or repeat), each of which lasted $12 \mathrm{~s}$ followed by $12 \mathrm{~s}$ of fixation. Participants performed a fixation color change detection task during each run. Each scene subtended $7.4^{\circ}$ and was presented centrally for $750 \mathrm{~ms}$ with an ISI of $1 \mathrm{~s}$. There were a total of four stimulus conditions for each run: familiar/indoor new, familiar/indoor repeat, unfamiliar/outdoor new, and unfamiliar/outdoor repeat. While viewing the scenes, subjects performed an orthogonal fixation color change detection task designed to encourage them to maintain fixation and attention throughout the experiment. The fixation cross, which appeared throughout each run, changed its color from black to red every $3-5 \mathrm{~s}$ during both the scene and fixation blocks. Participants were instructed to maintain fixation and to detect the color change with a button press.

Participants performed this task with high accuracy (control mean $=$ $95.7 \%$, J.N. $=98.5 \%$ for the familiar/unfamiliar scenes and control mean $=95.3 \%$, J.N. $=96.1 \%$ for the indoor/outdoor scenes). J.N.'s performance did not differ from that of the controls in either the familiar/unfamiliar scenes $(p=0.68)$ or in the indoor/ outdoor scenes ( $p=$ $0.75)$ experiments.

The motion parameters obtained from the preprocessing stages were used as nuisance variables in a regression model. The betas from this regression model (data not explained by motion) were used for the time course analyses. To quantify adaptation effects, an adaptation index (AI) was computed for each ROI for each subject (Pinsk et al., 2009) as follows:

$$
\mathrm{AI}=\frac{\text { Peak }_{\text {New }}-\text { Peak }_{\text {Repeat }}}{\sqrt{\left(\sigma_{\text {New }}^{2}+\sigma_{\text {Repeat }}^{2}\right) / 2}}
$$

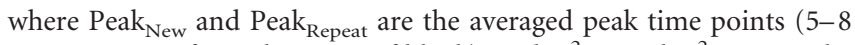
TRs or 8-16 s from the onset of block), and $\sigma_{\text {New }}^{2}$ and $\sigma_{\text {Repeat }}^{2}$ are the averaged variance of the peak responses for the new and repeat blocks, respectively. Although the condition blocks lasted $12 \mathrm{~s}$ ( 6 TRs total), to avoid potential contamination of the peak estimates as individual subjects' hemodynamic delay may vary, we used only the middle four peak TRs when computing AI values. For each subject, the AI was computed using average peak responses and variance across blocks. The AIs of the controls were then averaged to compare with J.N.'s AI. Positive AI values indicate that responses were greater for the new than repeat blocks. A one-way ANOVA was run to test for differences in AI values across ROIs.

In addition to the time course analysis, we also ran a general linear model (GLM) with square-wave functions matching the block design of the new and repeat conditions. This was done to compare AI results computed from the time course data and the adaptation effects as defined by new minus repeat in this regression model.

Familiar and unfamiliar scenes. Before the fMRI session, participants completed a landmark recognition survey that consisted of 80 colored photographs of famous landmarks across the world. Of the 80 landmarks, six were highly familiar and were from J.N.'s city of residence. Landmarks were labeled as familiar if the participant could either name (e.g., Notre Dame) and/or provide contextual information (e.g., a cathedral in Paris, France). On average, control participants recognized 37 landmarks and J.N. recognized 44. J.N. correctly recognized all six landmarks that are located in her home city, suggesting that J.N.'s navigation impairments lie within disorientation rather than place recognition. Because individuals differed in which landmarks they recognized, some scene stimuli were used for the familiar blocks in some participants, whereas the same stimuli were used for the unfamiliar blocks in others 
(32 exemplars in each category). The indoor and outdoor scenes consisted of the same set of independent and unfamiliar images for all participants (32 exemplars for each category).

Resting-state functional connectivity. Participants were run in $210 \mathrm{~min}$ resting-state runs in which they were instructed to rest and stare at a blank screen. For all participants, one of the resting-state runs was scanned at the beginning of the fMRI session to reduce "contamination" from seeing scenes in the subsequent runs. For each participant, WM and CSF masks were localized anatomically. The WM and CSF time courses and motion parameters were regressed out as nuisance variables. The voxel responses within an ROI were averaged and the resulting time course was used to temporally correlate across all pairwise ROIs using Pearson's correlation.

To quantify the correlation outputs to compare between seed and target ROIs, a normalized ranked correlation score was computed for each seed-to-target ROIs correlation. For a given seed region (e.g., RSC), the 12 target ROIs were ordered by the rank of their correlation (e.g., first $=$ PPA, second $=$ TOS, etc.). We then normalized by the total number of connections observed for that seed and subtracted from 1. A lower score indicates a lower connectivity between the seed and target ROI. Instead of comparing the raw correlation values, which may differ across individuals or across different seeds, the normalized connectivity score allows for a relative comparison of the seed-target correlation with respect to the seed's connectivity to the rest of the target ROIs examined. This normalized approach avoids any confounds associated with nuisance variables such as the potential inaccurate estimation of WM and CSF time courses or differences in the alertness of subjects during scans.

DTI analysis. Each subject was scanned in 1 DTI scan lasting $5.6 \mathrm{~min}$. The FSL Diffusion Toolbox (http://fsl.fmrib.ox.ac.uk/fsl/fslwiki/FDT) was used to preprocess the data for eddy current distortion and motion correction. Using the Bayesian Estimation of Diffusion Parameters Obtained using Sampling Techniques (BESPOSTX), diffusion tensors were fit for each voxel and the functional anisotropy and mean diffusion values were calculated for each voxel. The ROIs defined using functional localizers and FreeSurfer's automatic subcortical segmentation tool (HIP) were used as seeds in the probabilistic tractography (PROBTRAKX) tool to compute the connectivity distributions from each seed to every voxel of the brain. All of the preprocessing and tracking were done in the subject's native anatomical space. Subsequently, the tracking results were converted to MNI space using linear (FLIRT) and nonlinear (FNIRT) coregistration tools. To account for differences in ROI sizes that could result in different probabilistic tracking results, the path outputs were normalized by the RSC and PPA sizes (Rilling et al., 2008). The probabilistic tracking used 5000 samples/voxel, a curvature threshold of 0.2 , and was corrected for distance across the seed and target ROIs.

\section{Results}

\section{Behavioral results}

Comparison with other reported DTDs on navigational abilities We benchmarked J.N.'s performance using the Iaria and Barton (2010) online battery designed to test cognitive skills related to navigation. The battery includes nine subtests, some of which were designed for testing basic perceptual processes such as object recognition (subtest A), face identity recognition (subtest $\mathrm{B}$ ), face expression recognition (subtest $\mathrm{C}$ ), and landmark recognition (subtest D), and some of which focus directly on processes related to navigation such as heading orientation (subtest E), left/right orientation (subtest F), path reversal (subtest G), and formation (subtest $\mathrm{H}$ ) and use of (subtest I) a cognitive map. Compared with controls $(n=120)$, the DTD group $(n=120)$ reported in Iaria and Barton's (2010) study were only reliably different on subtests D-I. J.N.'s scores on all of the subtests were almost identical to the reported DTDs' scores, with a correlation of $r=0.92, p=0.001$. This result critically indicates that J.N.'s behavioral profile shows a specific impairment in navigation rather than in basic visual processing and closely matches that of other DTDs. Table 1 lists the results of J.N. and those of other
Table 1. Comparison of J.N.'s perceptual and navigation abilities with other DTDs and controls

\begin{tabular}{lllr}
\hline Subtest & $\begin{array}{l}\text { Control group } \\
(n=120)\end{array}$ & $\begin{array}{l}\text { DTD group } \\
(n=120)\end{array}$ & J.N. \\
\hline A. Object recognition & $99.8(0.01)$ & $99.2(0.06)$ & 100 \\
B. Identity recognition & $81.8(0.14)$ & $80.1(0.16)$ & 80 \\
C. Expression recognition & $96.5(0.06)$ & $94.6(0.10)$ & 100 \\
D. Landmark recognition* & $93.9(0.07)$ & $87.0(0.10)$ & 100 \\
E. Heading orientation* $^{*}$ & $88.3(0.13)$ & $77.8(0.17)$ & 60 \\
F. Left/right orientation* & $92.5(0.12)$ & $84.7(0.18)$ & 80 \\
G. Path reversed* & $83.5(0.17)$ & $70.1(0.22)$ & 50 \\
H. Formation of cognitive & $677(53)$ & $1311(0.53)$ & Not completed
\end{tabular}

$\mathrm{map}^{*}(\mathrm{RT}, \mathrm{ms})$

I. Use of cognitive map

$92.3(0.14)$

$71.7(0.26)$

40

J.N. completed an online perceptual battery developed by laria and Barton (2010) to assess navigation-related abilities (www.gettinglost.ca). This battery consists of nine subtests, which are designed to test either basic perceptual (A-D, e.g., view-invariant object and face recognition) or navigation skills (E-I, e.g., test of forming a cognitive map or testing for egocentric orientation judgment). A description of each subtest is reported in laria and Barton's report. J.N.'s performance on each task was compared with data from 120 individuals with DTD (mean age: 43.7, 102 females) and 120 controls (mean age: 42.3, 102 females) reported in laria and Barton's (2010) study. Each number denotes the percentage correct unless otherwise stated and SDs are noted in parentheses. Asterisks denote tests that yielded a significant difference in performance across the DTD group and the controls from laria and Barton's study. J.N.'s performance did not differ reliably from the DTD group's performance in any of the subtests. There was a high positive correlation between J.N. and the DTD group's performances across eight subtests, excluding the formation of a cognitive map task (H), which J.N. was not able to complete due to high difficulty. $r=0.92, p=0.001$.

DTD individuals and controls published in Iaria and Barton's (2010) study.

\section{Santa Barbara Sense of Direction Scale}

The Santa Barbara Sense of Direction Scale (SBSDS; Hegarty et al., 2002) is a 15-item survey measuring self-reported spatial and navigational abilities, preferences, and experiences. The SBSDS has high test-retest reliability (Kozhevnikov and Hegarty, 2001) and scores on this scale are highly predictive of one's ability to update one's orientation, path integration, and computation of location and heading and to learn one's location relative to landmarks (Hegarty et al., 2002; Sholl et al., 2006). As expected, J.N. scored dramatically poorly on this questionnaire, with a score of 18.0 ( $>4$ SDs from the control mean). Controls scored a mean of 78.0 (SD: $12.3, p=0.006$ ). The eight controls who participated in the MRI experiments also completed the SBSDS and their mean score was $65.0(\mathrm{SD}=12.9)$, reliably higher than that of J.N. at $18.0(p=0.01)$.

\section{Card rotation test and paper-folding test}

J.N. performed marginally poorly on both tests designed to test spatial imagery and mental manipulation of spatial information (Ekstrom et al., 1976). On the card rotation test, she scored 28.0, which was poorer than the controls' mean $=53.7(\mathrm{SD}=11.4, p=$ $0.09)$. On the paper-folding test, she scored a 1.6, whereas the control participants scored a mean of $6.0(\mathrm{SD}=2.1, p=0.11)$.

\section{Mental rotation test}

To assay further J.N.'s ability to process spatial information mentally, we devised a mental rotation test similar to that of Podzebenko et al. (2002) in which participants determined whether an individually displayed asymmetric letter or digit (e.g., "5") was in the standard or mirror-reversed view. In blocks of trials, the stimulus rotated in plane by the following degrees: $0,15,30,80$, and 95. Although J.N.'s accuracy in the task was comparable to that of controls (J.N. $=96.3 \%$ vs controls $=98.2 \%, \mathrm{SD}=1.2 \%$ ), J.N.'s overall reaction time (RT) (994.4 ms, correct trials only) was significantly slower than controls' RT (645.9 ms, SD = 59.9 ms), $p=0.003$. Consistent with previous studies in mental rotation (Podzebenko et al., 2002), both J.N. and the controls' RTs increased in a linear fashion with increasing degrees of stimulus 
rotation (J.N.'s linear fit: $R^{2}=0.99$ vs controls linear fit: $R^{2}=$ $0.92, \mathrm{SD}=0.13$, comparison between J.N. and controls' linear fits, $p=0.68$ ). Despite this, J.N.'s RT had a significantly steeper slope of 6.9 compared with controls' slope of 1.7 ( $\mathrm{SD}=0.9, p=$ 0.003 ). Consistent with the card rotation and paper-folding tasks, J.N. showed greater difficulty than controls in tasks requiring mental rotation and spatial manipulation, implicating impairment in working with and manipulating spatial information. These findings suggest that the deficit extends beyond spatial processing within the external environment and affects internal spatial representations and processes as well.

\section{Heading orientation test}

To assess heading orientation, J.N. performed a task developed by Hashimoto and colleagues (2010), in which she stood within the center of a $3 \times 3$ grid on the floor and was required to remember the location of 3 cards with different shapes placed in different cells of the grid (study phase). After the cards were removed, J.N. was instructed to place the cards back on the grid (test phase). In Part A of the task, J.N. remained in the same position during the study and test phases, but in Part B, she was required to physically rotate her body either $90^{\circ}$ or $180^{\circ}$ clockwise or counterclockwise during the interval between the study and test phases. J.N.'s score for Part A was slightly worse than controls' (J.N. = 24.0, controls $=27.8, \mathrm{SD}=1.6, p=0.08)$ and J.N.'s score for Part B was reliably worse than controls' (J.N. $=17.0$, controls $=26.7, \mathrm{SD}=$ 2.9, $p<0.03$ ). Interestingly, J.N.'s performance did not differ reliably from the retrosplenial topographic patients from Hashimoto et al.'s (2010) study (comparison between J.N.'s and patients' scores: Part A, patient mean $=26.7, \mathrm{SD}=1.2, p=0.18$; Part $\mathrm{B}$, patient mean $=11.0, \mathrm{SD}=6.6, p=0.51$ ), suggesting that, notwithstanding the absence of a frank restrosplenial lesion, J.N.'s difficulty updating spatial information resembles that of the retrosplenial patients.

\section{Face, object, and scene recognition tests}

J.N. performed with high accuracy on both the recognition of famous American faces (Avidan et al., 2005), with a score of $98.2 \%$ (controls $=88.7 \%, \mathrm{SD}=4.9 \%, p=0.13$ ), and the Cambridge Face Memory Test (Duchaine and Nakayama, 2006) (J.N. = $79.2 \%$, controls $=81.7 \%, \mathrm{SD}=14.9 \%, p=0.88)$. On an object recognition test with 60 common items (Behrmann and Kimchi, 2003), J.N. performed $96.7 \%$, which was comparable to that of controls, $($ mean $=98.2 \%, \mathrm{SD}=1.1 \%, p=0.25)$.

Two tests of scene classification were devised. The first test was an easy indoor or outdoor judgment task performed on 32 photographs of different scenes: 16 indoor scenes (e.g., bedrooms, dining rooms, foyers, etc.) and 16 outdoor scenes (e.g., building exteriors, courtyards, pools, etc.). Each scene was presented for $500 \mathrm{~ms}$ and the participant had up to $3 \mathrm{~s}$ to respond. The second test was a slightly more difficult indoor scene classification test in which photographs of scenes from one of four categories were presented (i.e., kitchen, office, bedroom, or living room) with 15 exemplars of each category. Scenes were presented for up to $3 \mathrm{~s}$ and participants were required to name aloud the scene category of the exemplar as quickly and accurately as possible. None of the scene stimuli used in these behavioral tests was used in any of the fMRI investigations or in the familiar landmark recognition questionnaire described above. J.N.'s performance was consistently high and comparable to that of controls. She scored $100 \%$ in the indoor/outdoor test (controls, 96.4\%, $\mathrm{SD}=3.9 \%, p=$ 0.44 ) and her RT (1091 ms) was equivalent to that of controls (925 ms, SD $=176 \mathrm{~ms}, p=0.42$ ). The second indoor scene classification task replicated these findings: J.N. scored $97.5 \%$ versus controls' mean score of $99.0 \%(\mathrm{SD}=1.0, p=0.21)$ and her RT (1020 ms) was also comparable to that of controls (1139 $\mathrm{ms}, \mathrm{SD}=89 \mathrm{~ms}, p=0.27)$. As noted in the Materials and Methods section, a famous landmark questionnaire was also administered to determine the familiar and unfamiliar scenes to use in the fMRI experiment. J.N.'s performance was comparable to controls. Of the landmarks assessed, six were personally familiar to J.N. because they were within her home city. J.N. was able to identify all of them. Together, these results clearly indicate that J.N.'s topographic disorientation cannot be explained by a compromise in visual form perception or in scene recognition per se.

\section{Item memory tests}

J.N. performed within normal limits on the Wechsler Memory Scale (Wechsler, 1997) for verbal, digit, and spatial memory; her performance on remembering word lists and paired associates achieved normed scores between 8 and 14. On these normed scales, scores 8-12 are considered average and scores 13-14 high average. Controls scored similarly to J.N. and there was no difference between J.N. and the controls in the word list $(p=0.21)$ or paired associates tasks $(p=0.60)$. J.N.'s performance on the forward digit span was 10 , which was comparable to controls (mean $=10.8, \mathrm{SD}=2.1, p=0.73$ ). Her performance on the forward spatial span assessing visuospatial working memory, however, fell in the low average range, achieving a raw and normed score of 6 (scores of 6-7 are considered low average). Controls' score was $9.5(\mathrm{SD}=1.0)$ and this was reliably higher than that of J.N.'s $(p=0.03)$.

Together, these behavioral results indicate that J.N. has deficits in representing, updating, and using spatial information (i.e., card rotation, paper folding, card placement, mental rotation, and visuospatial working memory) without a general nonspatial memory problem (i.e., normal performance on the Wechsler Memory verbal and digit subtests and Cambridge Face Memory Test) or a deficit in visual form perception (i.e., normal performance on object and face recognition and scene classification tests). These results are corroborated by her profile on the DTD online battery from Iaria and Barton's (2010) study, as described above.

\section{Functional localizer}

A comparison of the functionally defined scene and early visual areas in J.N. and in a representative control subject, C1, is shown in Figure $1 B$. Despite her navigational deficits, we were able to functionally localize scene-selective ROIs showing greater BOLD response to scenes than other object classes. The amplitude of J.N.'s scene responses in PPA, TOS, and RSC (Fig. 2) did not differ from that of controls (all $t<1.00, p>0.40$ ). In controls, consistent with previous accounts of a right hemisphere bias of scene selectivity in these regions (Epstein et al., 2003), there were greater scene-selective responses in the right than left PPA $\left.t_{(12)}=6.48, p<0.0001\right)$ and right than left RSC $\left(t_{(12)}=4.68, p=\right.$ $0.0005)$. There was no hemispheric difference in TOS in controls $\left(t_{(12)}<1.00, p=0.52\right)$. J.N. also showed right hemisphere dominance for scene selectivity in PPA and RSC and no bias in TOS. The magnitude of the right hemisphere bias was indistinguishable from that of controls (PPA: $p=0.48$; RSC: $p=0.80$, TOS: $p=0.74)$. J.N.'s scene ROI size $(0.18<p<0.95)$ and cortical thickness $(0.19<p<0.88)$ also did not differ reliably from controls' ROIs.

Similar to the scene ROIs, the amplitude of responses to objects in LOC and to faces in FFA were comparable across J.N. and 
A

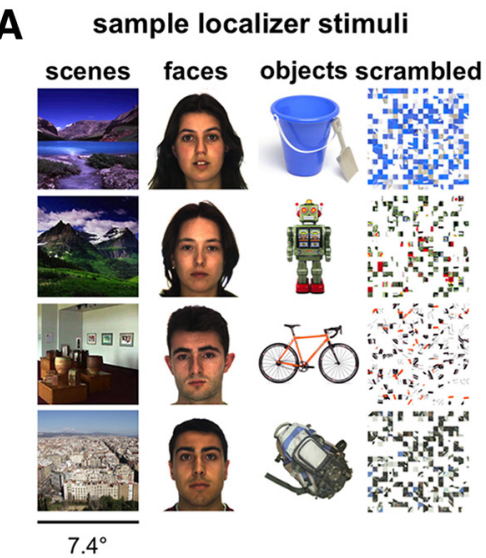

$7.4^{\circ}$
B scene ROIs in JN

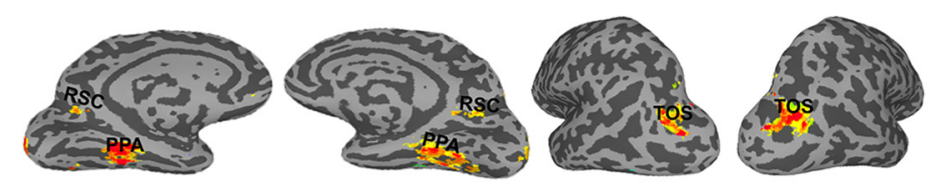

scene ROls in C1

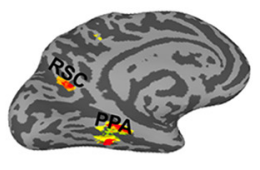

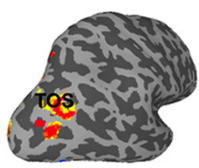

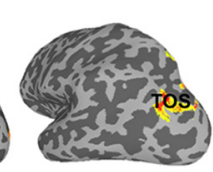

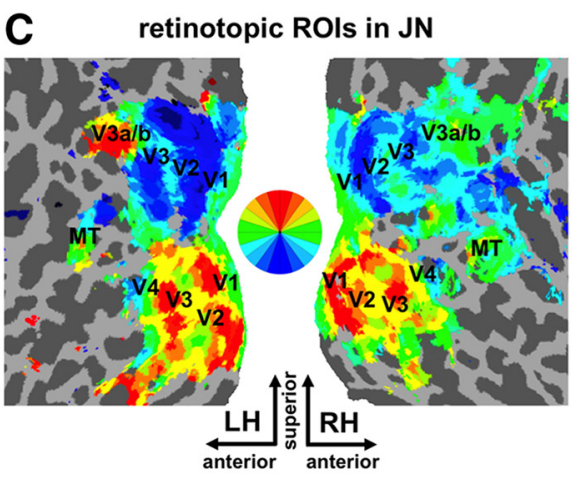

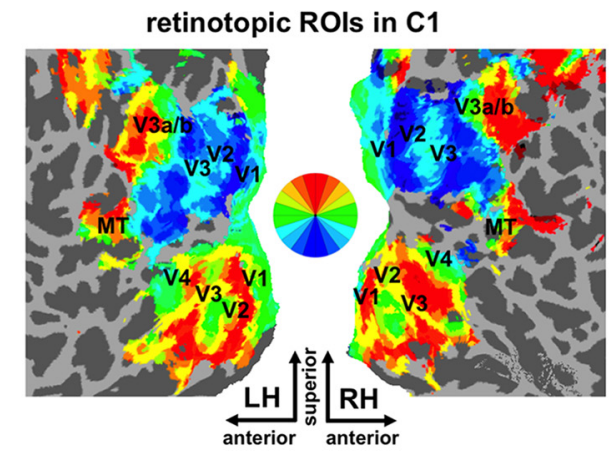

Figure 1. Sample stimuli used for functional localizers: scene and topographic ROIs in J.N. and control subject C1.A, Sample stimuli used for functional localizers. B, Scene-selective RSC, PPA, and TOS in J.N. and C1. C, Topographically organized ROIs in visual cortex including V1, V2, V3, V4, MT, V3a, and V3b are shown on flattened surface reconstructions in J.N. and C1. Retinotopic mapping revealed patterns of topographic organization in both hemispheres in J.N. that were similar to controls.

controls (both regions $p>0.48$ ). J.N.'s early visual areas showed topographic organization that was similar to controls'. There was no reliable difference in size (all $p>0.45$ ) or cortical thickness (all $p>0.63$ ) between J.N. and the controls in any of the nonscene-selective ROIs, including the HIP, LOC, FFA, and retinotopic areas.

The results from the functional localizer experiment demonstrate that J.N. has identifiable regions of cortex that show appropriate category-selective responses and that there is no reliable difference in any of the dependent measures extracted between J.N. and controls.

\section{Adaptation experiments}

One common functional property of ROIs is that the mean BOLD response to the presentation of repeated identical stimuli is markedly reduced compared with that of a sequence of different stimuli. The examination of this adaptation effect (i.e., repetition suppression) has been used successfully to uncover the selectivity of representations in scene-selective ROIs (Epstein et al., 2008; Epstein and Morgan, 2012; Ward et al., 2013). Because J.N.'s deficit might arise from an alteration in the nature of the representations in scene ROIs, we investigated whether she shows the expected adaptation effect in two independent experiments. The first experiment used familiar and unfamiliar scenes of famous landmarks that were preselected for each individual participant before scanning. The second experiment used indoor and outdoor scenes (Fig. $3 A, B$ ) that were unfamiliar to all participants (see Materials and Methods).

Figure $3 C$ shows the time course for responses in PPA, TOS, and RSC for J.N., C1, and averaged controls. The AIs, which were calculated to quantify the response differences between the new and repeat conditions, are shown in Figure $4 A$. The left and right hemispheres showed a similar pattern of results (Fig. 4B) and only the aggregated bilateral results are reported here. Controls showed reliable AIs for both familiar/unfamiliar scenes in PPA $\left(t_{(12)}=9.75, p<0.00001\right)$, TOS $\left(t_{(12)}=7.73, p<0.00001\right)$, and $\operatorname{RSC}\left(t_{(12)}=9.19, p<0.00001\right)$ and for indoor/outdoor scenes in $\operatorname{PPA}\left(t_{(12)}=8.49, p<0.00001\right)$, TOS $\left(t_{(12)}=6.71, p<0.00001\right)$, and RSC $\left(t_{(12)}=8.96, p=0.00001\right)$. Similarly, J.N. showed positive AIs in PPA and TOS. Although the AIs for J.N.'s PPA were numerically reduced compared with those of the averaged controls', they were not reliably different for either familiar/unfamiliar scenes $\left(t_{(12)}=-1.16, p=0.27\right)$ or indoor/outdoor scenes $\left(t_{(12)}=-1.14, p=0.28\right)$. J.N.'s TOS AIs also did not differ from controls' for either familiar/unfamiliar $\left(t_{(12)}<1.00, p=0.61\right)$ or indoor/outdoor scenes $\left(t_{(12)}<1.00, p=0.92\right)$. In contrast to controls, however, J.N.'s RSC did not evince differential responses to the new versus repeat blocks and the AIs were markedly reduced relative to controls for both familiar/unfamiliar $\left.t_{(12)}=-2.31, p=0.04\right)$ and indoor/outdoor scenes $\left(t_{(12)}=\right.$ $-2.38, p=0.03)$. Consistent with the results from the localizer experiment (Fig. 2A), the amplitude of the peak responses in J.N.'s RSC (collapsed across conditions) did not differ reliably from controls' RSC for both the familiar/unfamiliar scenes $\left(t_{(12)}<1.00, p=0.48\right)$ and indoor/outdoor scenes $\left(t_{(12)}<1.00\right.$, $p=0.52)$. Therefore, whereas J.N.'s RSC is responsive to scenes, it does not differentiate between different and identical scenes.

For controls, there were significant differences between AI values across ROIs (PPA, TOS, and RSC), for familiar/unfamiliar scenes $\left(F_{(2,36)}=7.94, p=0.001\right)$ and indoor/outdoor scenes 
category selective responses

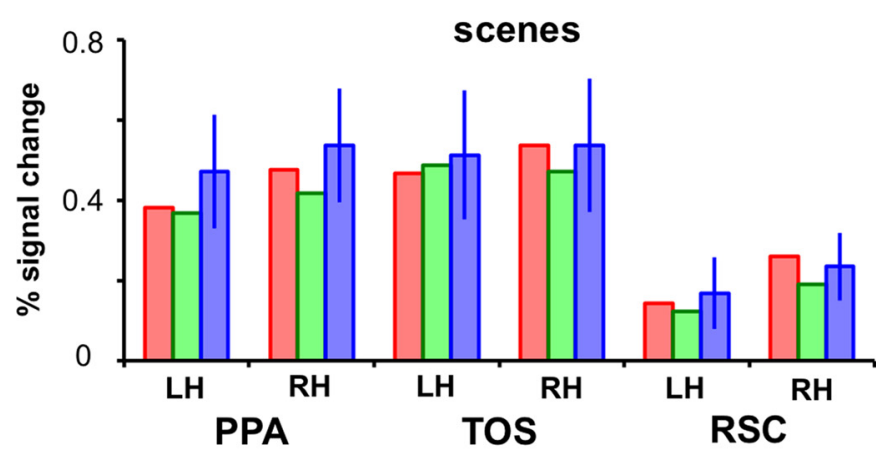

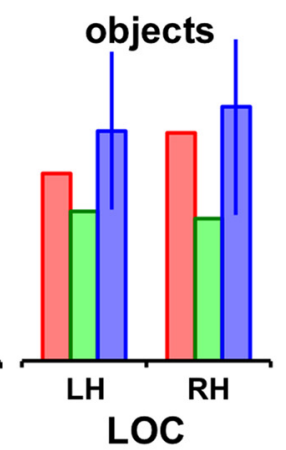

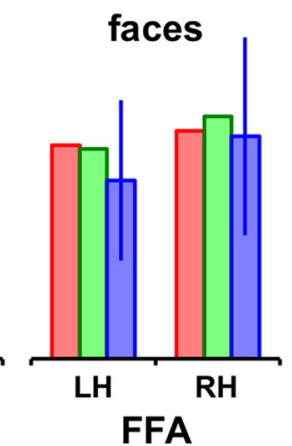

\section{ROI size}

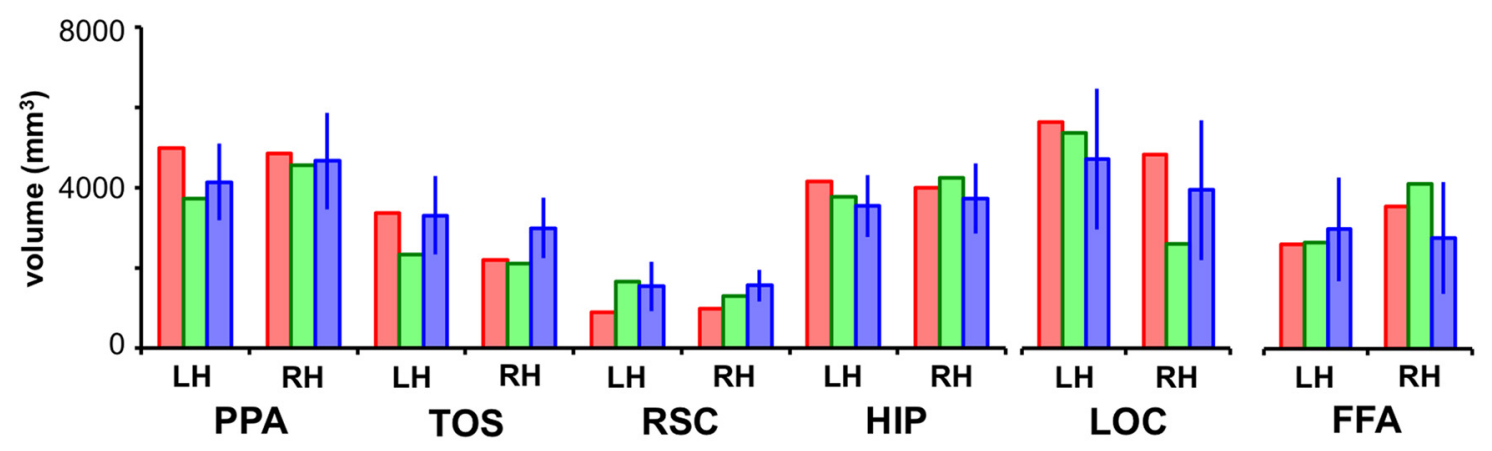

cortical thickness

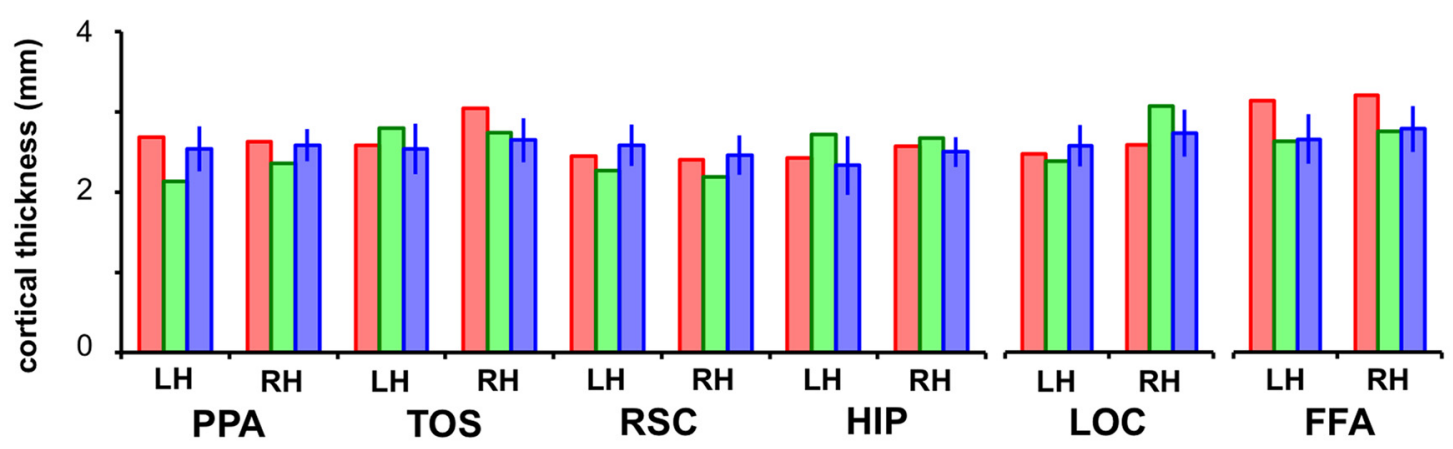

Figure 2. Category-selective responses, ROI sizes, and cortical thickness in J.N. and controls. Scene-selective responses in J.N.'s PPA, TOS, and RSC were not reliably different from control subjects in both hemispheres. Similarly, object-selective responses in J.N.'s LOC and face-selective responses in J.N.'s FFA were not reliably different from that in controls. None of the ROIs examined were different in size or cortical thickness between J.N. and controls. Error bars indicate SD.

$\left(F_{(2,36)}=7.00, p=0.003\right)$. Post hoc tests with Bonferroni correction showed that AIs in PPA $(p=0.001$ for familiar/unfamiliar and $p=0.003$ for indoor/outdoor $)$ and TOS ( $p=0.04$ for familiar/unfamiliar and $p=0.03$ for indoor/outdoor) were significantly higher than in RSC. The PPA-RSC and TOS-RSC difference in AI scores for J.N. was not reliably different from that of controls for both familiar/unfamiliar and indoor/outdoor scenes $(0.29<p<0.95)$.

Results from the GLM in which new and repeat conditions were modeled showed a similar pattern of results to the AI findings. Controls showed reliable adaptation effects as defined by a positive new minus repeat $\beta$ difference for familiar/unfamiliar scenes (PPA: $t_{(12)}=12.77, p<0.00001$, TOS: $t_{(12)}=13.20, p<$ 0.00001 , and RSC: $\left.t_{(12)}=8.51, p<0.00001\right)$ and indoor/outdoor scenes $\left(\mathrm{PPA}: t_{(12)}=11.49, p<0.00001\right.$, TOS: $t_{(12)}=9.15, p<$
0.00001, and RSC: $\left.t_{(12)}=10.08, p<0.00001\right)$. J.N. also showed a positive new minus repeat difference for PPA and TOS that was not reliably different from that of controls (all $p>0.14$ ). Critically, J.N.'s RSC showed significantly reduced adaptation effect compared with controls for both familiar/unfamiliar scenes $\left(t_{(12)}=-2.34, p=0.04\right)$ and indoor/outdoor scenes $\left(t_{(12)}=\right.$ $-2.42, p=0.03)$.

To test for differences in the responses to the different scene types (familiar $>$ unfamiliar or indoor $>$ outdoor), similar quantification as for the AI quantification was used. In this method, the difference in average peak responses to the different scene types was examined as a proportion of the variances from the scene types (Fig. 4C). Consistent with previous reports on the effect of familiarity in scene ROIs (Cabeza et al., 2004; Epstein et al., 2007a), all scene ROIs in controls showed greater BOLD re- 
A

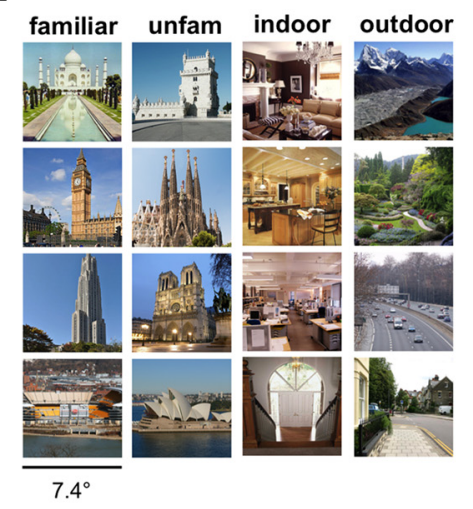

C JN's scene ROIs

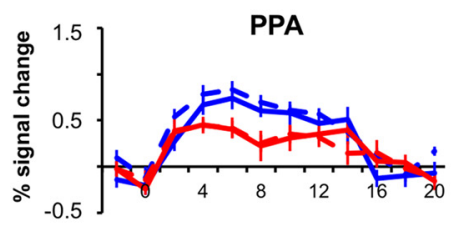

C1's scene ROIs

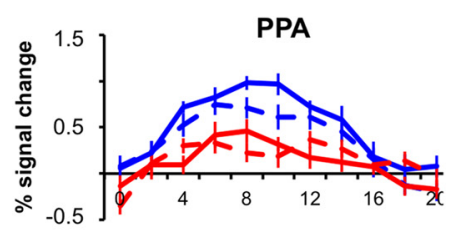

averaged controls' scene ROls

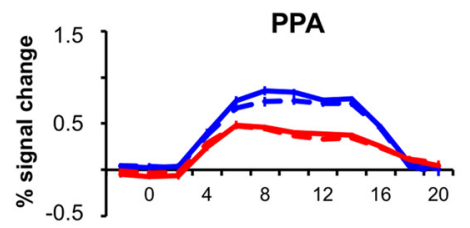

B

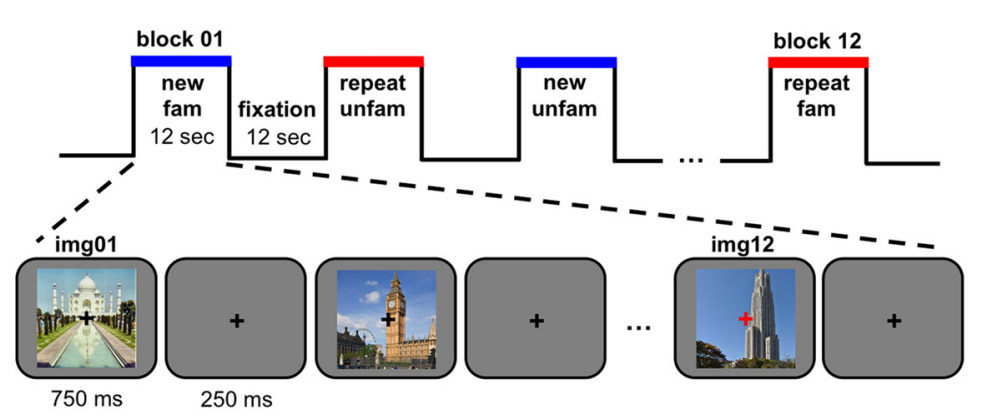

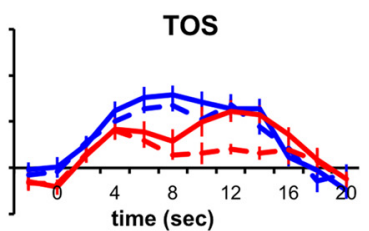
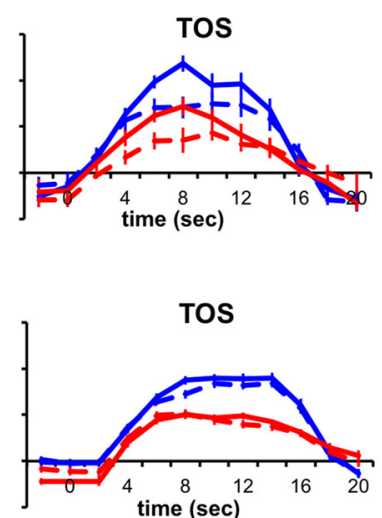

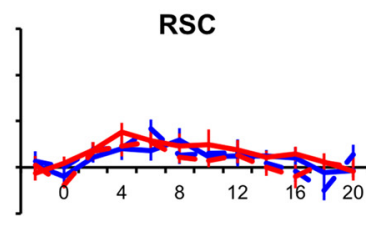

RSC

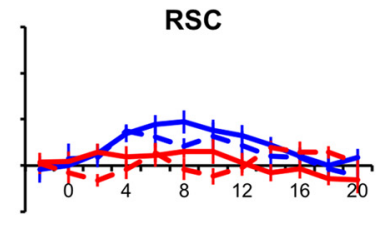

RSC

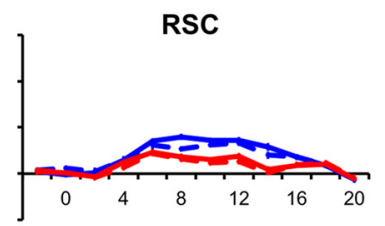

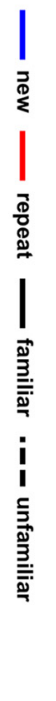

Figure 3. Sample stimuli, presentation sequence, and time course results for the adaptation experiment. $\boldsymbol{A}$, Sample scenes used for the adaptation experiments. The familiar and unfamiliar scenes shown are those that were familiar and unfamiliar to J.N. B, Presentation sequence of the adaptation experiments. During scene and fixation blocks, subjects were instructed to detect a fixation color change. C, Time courses of responses from PPA, TOS, and RSC from the familiar/unfamiliar adaptation experiment are shown for J.N., $C 1$, and averaged controls. Blue lines indicate new scenes and red lines indicate repeated scenes. Solid lines indicate scenes that were familiar and dotted lines scenes that were unfamiliar.

sponses to familiar than unfamiliar scenes (PPA: $t_{(12)}=4.14, p=$ 0.001 , TOS: $t_{(12)}=2.92, p=0.01$, RSC: $\left.t_{(12)}=2.29, p=0.04\right)$. The familiarity effects were also observed in J.N.'s ROIs and the magnitude of these effects were not reliably different from controls in PPA $\left(t_{(12)}<1.00, p=0.48\right)$ and $\operatorname{RSC}\left(t_{(12)}<1.00, p=\right.$ $0.79)$. Surprisingly, J.N.'s TOS showed a higher magnitude of the familiarity effect than controls' TOS $\left(t_{(12)}=3.01, p=0.01\right)$.

None of the controls' scene ROIs demonstrated differential BOLD responses to indoor versus outdoor scenes $\left(\mathrm{PPA}, t_{(12)}=\right.$ $1.08, p=0.30$, TOS, $t_{(12)}<1.00, p=0.55$ and RSC, $t_{(12)}<1.00$, $p=0.78$ ). The same was true for J.N. and controls (PPA, $t_{(12)}<$ $1.00, p=0.73$, TOS, $t_{(12)}=-1.04, p=0.32$, and RSC, $t_{(12)}<$ $1.00, p=0.50)$.

\section{Functional connectivity}

Given that J.N.'s RSC does not reveal a typical response profile, the question is whether this perturbation is limited to the RSC or if it might also be evident in the effective connectivity to other cortical regions. To test this, we examined the functional connectivity (i.e., temporal correlation) of 13 ROIs (V1, V2, V3, V4, V3a,
V3b, MT, LOC, FFA, PPA, TOS, RSC, and HIP) while participants were at rest-that is, with no visual stimulation or active task condition-so as to rule out the potential time-locked response changes due to evoked activity that can influence the resulting correlation values. For each participant, a symmetric $13 \times$ 13 correlation matrix was computed containing a cell for each pair of ROIs. Figure $5 A$ shows the correlation matrix for J.N., C1, and the average controls. In controls, there were high functional correlations across regions within the scene network. In J.N., however, the correlations between the scene ROIs were reduced. To quantify this, for each subject, we calculated the normalized ranked connectivity scores (range $0-1$ ) for each ROI as a seed region. A higher score indicates that the responses from the target ROI were more highly correlated with the seed region than other target ROIs.

In examining RSC's correlation profile to the other ROIs, the PPA was ranked either first or second highest in all individual controls with an averaged connectivity score of 0.89 ( $\mathrm{SD}=0.06$; Fig. $5 B$ ). In J.N., however, PPA was ranked second to the lowest with a connectivity score of 0.08 (the last being FFA). This differ- 
A

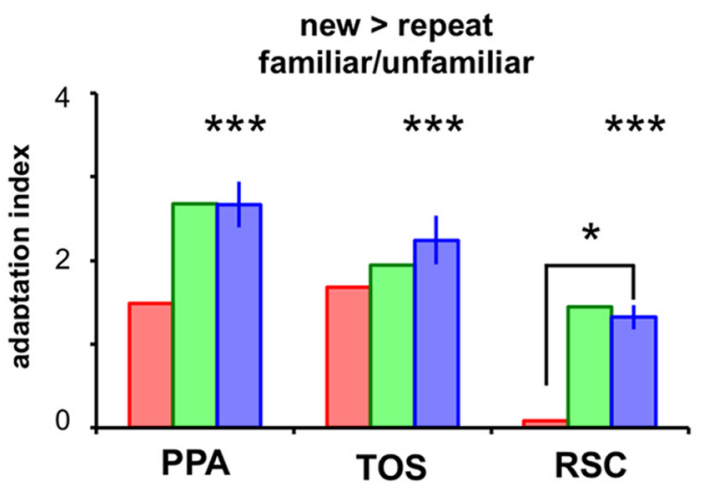

B

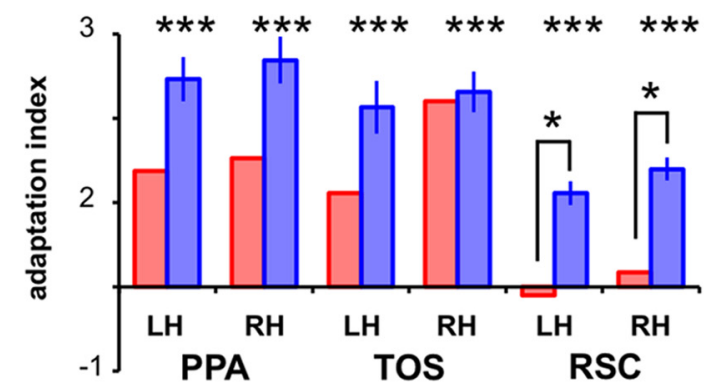

C

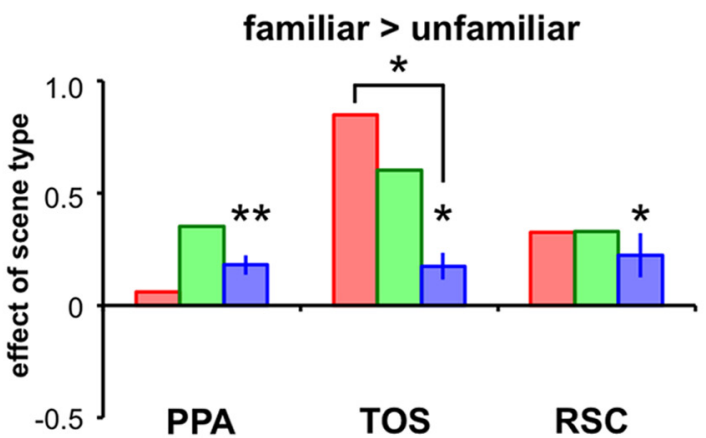

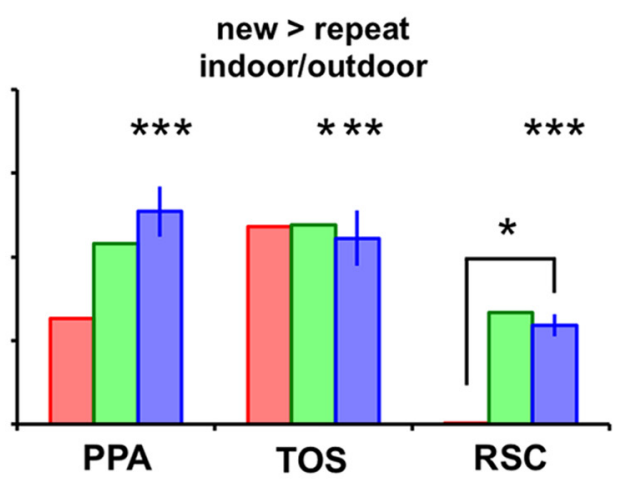

indoor/outdoor scenes
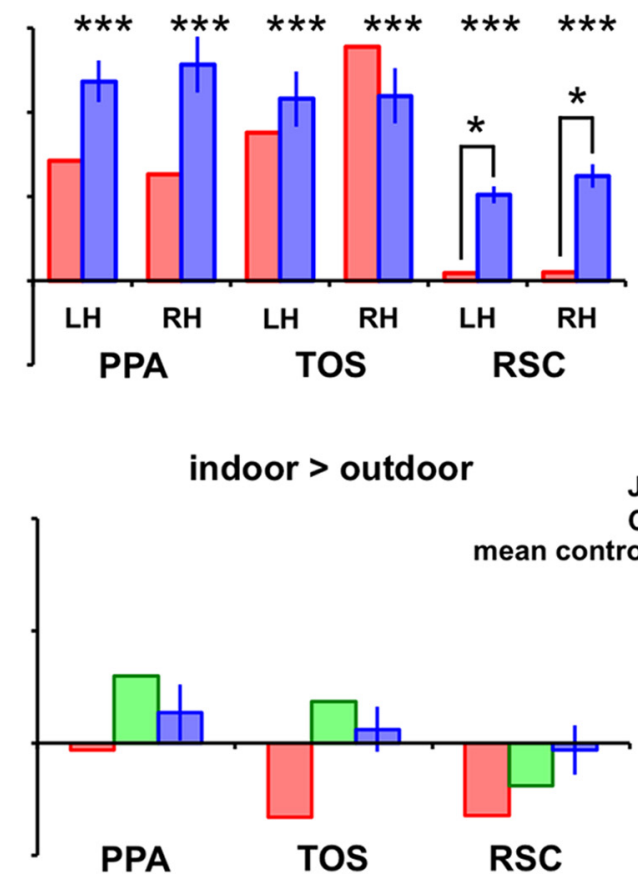

Figure 4. Al and effect of scene types. $A$, Across all scene ROls and experiments, controls showed reliable adaptation effects (asterisks above blue bars, ${ }^{* * *} p<0.001$ ). Error bars indicate SEM to assess the reliability of adaptation effects (AI >0). J.N.'s Als were not reliably different from controls in PPA and TOS. However, for both experiments, J.N.'s RSC did not show adaptation effects (asterisks comparing J.N. vs controls, ${ }^{*} p<0.05$ ). B, Als are shown separately for each hemisphere in both experiments. Compared with controls, J.N.'s RSC in both hemispheres consistently showed reduced Als for both familiar/unfamiliar scenes and indoor/outdoor scenes. C, Both controls and J.N. showed a reliable familiarity effect (familiar $>$ unfamiliar, ** $p<0.01$ ) in PPA, TOS, and RSC. None of the ROIs showed an effect for indoor versus outdoor scenes.

ence in the correlation scores of RSC to PPA between J.N. and controls was highly significant $\left(t_{(12)}=-12.46, p<0.0001\right)$. There were no reliable hemispheric differences in controls' RSC to PPA connectivity scores $\left(t_{(12)}<1.0, p=0.60\right)$ and both J.N.'s left $\left(t_{(12)}=-5.39, p<0.0001\right)$ and right $\left(t_{(7)}=-4.755, p=\right.$ $0.0001)$ RSC to PPA connectivity scores were significantly reduced compared with controls' (Fig. 6A).

When PPA was seeded, J.N.'s TOS connectivity score was 0.08 compared with controls' score of $0.65\left(\mathrm{SD}=0.25, t_{(12)}=-2.124\right.$, $p=0.06)$. Other seed-target connectivity patterns in which RSC, PPA, TOS, and HIP were used as seeds and targets did not show reliable differences across J.N. and controls $(0.13<p<0.98)$. Although only marginally reliable in PPA to TOS, J.N.'s scene ROIs may not be as functionally coupled as those in controls; this was most evident in the connectivity profile of RSC to PPA.

To test whether J.N.'s scene areas have deviant connectivity profiles to areas outside the scene network, we compared scene
ROIs' connectivity with a control region, the LOC. When RSC was seeded, J.N.'s LOC resulted in a score of 0.17 , which was well within the normal range (mean $=0.37, \mathrm{SD}=0.23, t_{(12)}=$ $-0.851, p=0.41)$. Similarly, when PPA was seeded, J.N.'s LOC score (0.42) was comparable to controls' LOC (mean $=0.56$, $\left.\mathrm{SD}=0.22, t_{(12)}=-0.623, p=0.55\right)$. Last, when TOS was seeded, J.N.'s LOC (0.75) showed similar rank as controls' LOC (mean = $\left.0.72, \mathrm{SD}=0.13, t_{(12)}=0.20, p=0.85\right)$.

The patterns of the functional connectivity structure are clearly visible in the ROI arrangements in Figure $5 C$. Here, an unsupervised data-driven hierarchical clustering approach was used to link similar resting-state correlation patterns across different ROIs. The hierarchical clustering analysis (Johnson, 1967) assumes the existence of some grouping structure without assuming particular grouping categories and links similar restingstate correlation patterns across different ROIs. The value of the $y$-axis denotes the response dissimilarity $(1-r)$, so the shorter 
A

JN

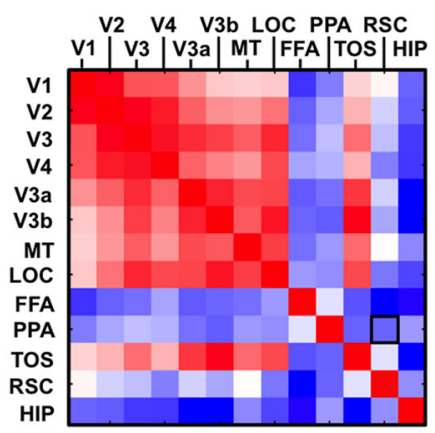

C1

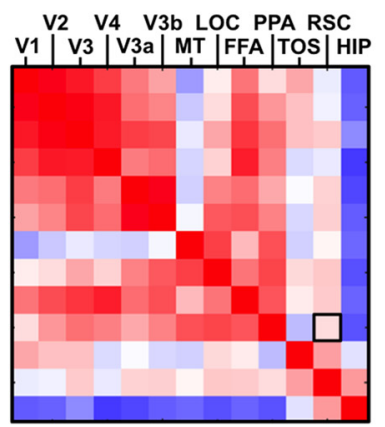

mean controls

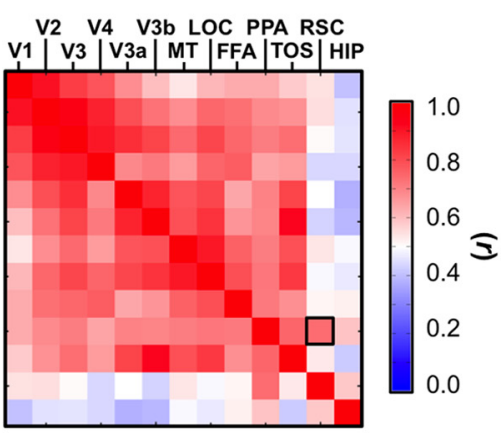

B
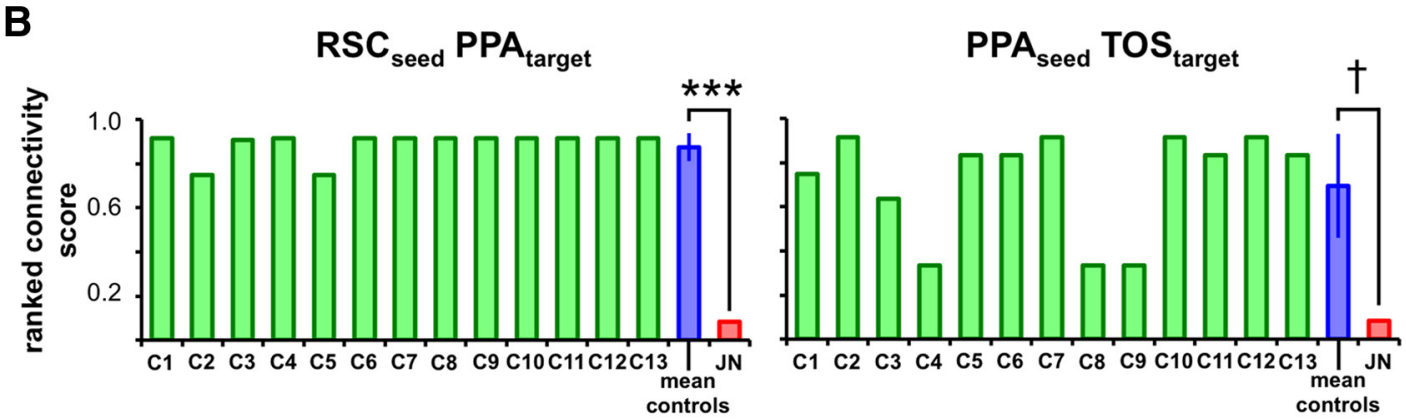

C
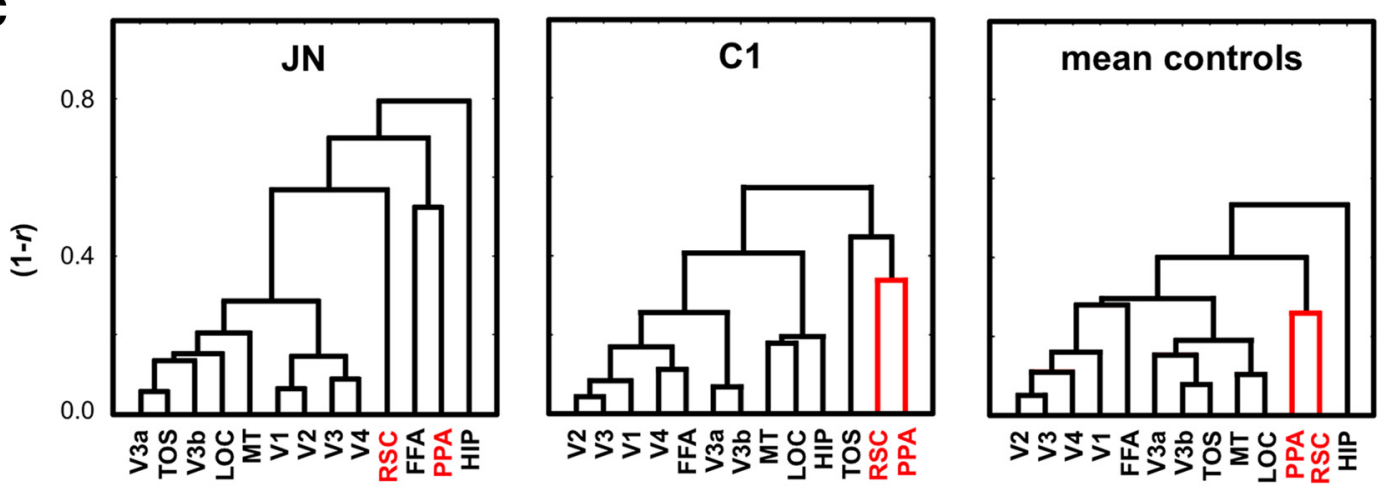

Figure 5. Functional connectivity. A, Temporal correlation matrix for J.N., C1, and averaged controls. B, The connectivity scores shown for individual (C1-C13) and averaged controls and J.N. across different seed and target ROIs. When RSC is seeded, J.N.'s PPA resulted in a significantly lower correlation than controls' $\left.{ }^{* * *} p<0.0001\right)$. When PPA was seeded, J.N.'s RSC and T0S resulted in lower connectivity scores compared with controls' $(\dagger p=0.06)$. C, Hierarchical clustering results show that J.N.'s RSC is not clustered with PPA.

height of the horizontal link indicates a reduced dissimilarity (or greater similarity) in the connectivity profiles of the ROIs linked. This analysis revealed a tight clustering of RSC and PPA in controls (Fig. 5C, red). J.N.'s RSC, however, emerged as an island on its own, not being linked to any other ROIs. Despite these differences in the scene ROIs, some similarities between J.N.'s and controls' functional connectivity results are evident. Consistent with the controls' data, J.N.'s early visual areas (e.g., V1-V4) are tightly clustered to each other and J.N.'s TOS showed high connectivity to V3a and V3b. TOS, V3a, and V3b are anatomically adjacent to each other and it is likely that TOS encodes more low-level visual features contained in scenes than do either PPA or RSC (Hasson et al., 2003; Dilks et al., 2013).

To test whether the reduced functional connectivity between J.N.'s RSC and PPA was evident under other conditions as well, we further examined the background functional connectivity (Norman-Haignere et al., 2012) of RSC and PPA during scene perception. To do so, we used data from the adaptation experiments in which evoked activity from stimuli presentations were regressed out as nuisance variables and the residual BOLD patterns were used as inputs for the correlation analysis (Al-Aidroos et al., 2012). The data from the adaptation experiments resulted in an identical pattern of results to the resting-state data analysis (Fig. 6B). When RSC was seeded, J.N.'s PPA resulted in a consistently lower connectivity score compared with control subjects' PPA scores. This result held with both the familiar/unfamiliar scenes data (J.N.'s score of $<0.01$ and controls' score of 0.79 , $\left.\mathrm{SD}=0.13, t_{(12)}=-5.75, p<0.0001\right)$ and the indoor/outdoor scenes data (J.N.'s score of 0.08 and controls' score of $0.81, \mathrm{SD}=$ $\left.0.16, t_{(12)}=-4.44, p=0.001\right)$.

Although highly unlikely, it is possible that, when J.N.'s RSC is seeded, there are regions just outside of the functionally defined PPA that show reliable correlation to RSC responses. Similarly, when PPA is seeded, it is possible that there are voxels in the vicinity of the functionally defined RSC that show correlation to PPA responses. To test this, we ran a whole-brain analysis to search for those voxels that were correlated with RSC and PPA resting-state responses. Results for J.N. and C1 are shown in Fig- 
A

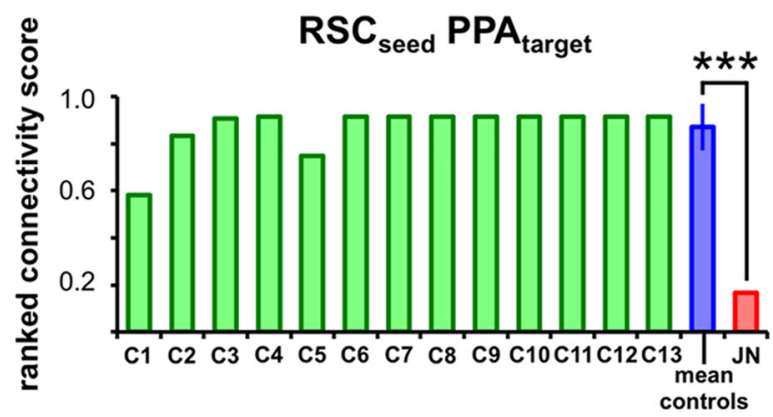

B

familiar/unfamiliar adaptation exp

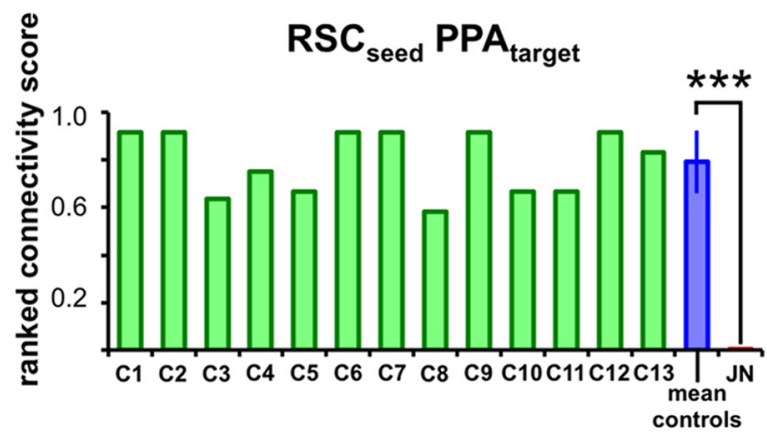

right hemisphere

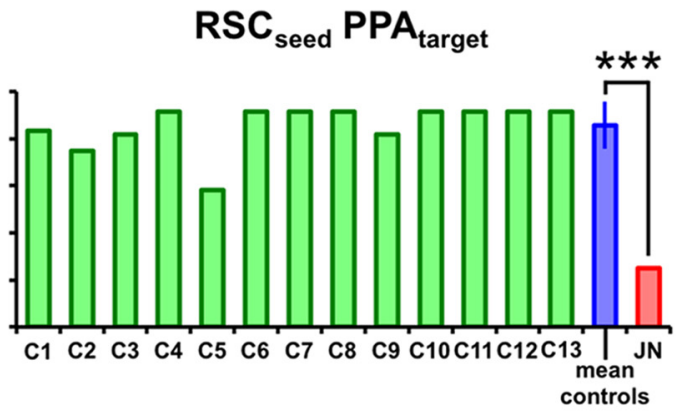

indoor/outdoor adaptation exp

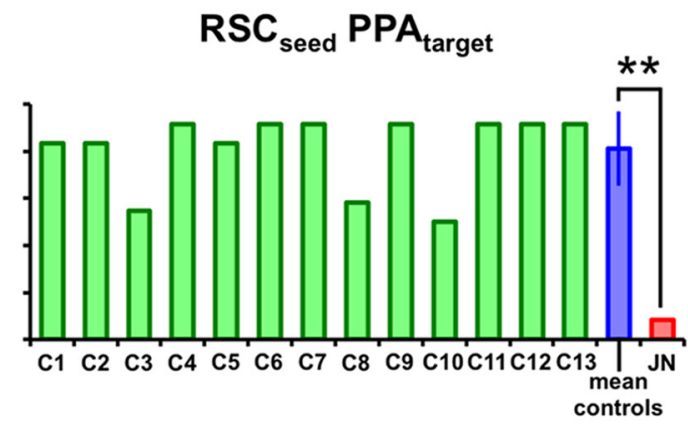

Figure 6. Functional connectivity results for left and right hemispheres and adaptation experiments. $A$, The ranked connectivity scores for each control subject (C1-C13), averaged controls, and J.N. are shown for the left and right hemispheres with RSC as the seed and PPA as the target ROI using the resting-state data. $\boldsymbol{B}$, Bilateral functional connectivity scores using the adaptation experiments data. J.N.'s RSC-PPA connectivity scores were significantly reduced compared with that of controls in all analyses $\left({ }^{* *} p<0.01,{ }^{* * *} p<0.001\right)$.

ure 7. When J.N.'s RSC was seeded and we examined regions near J.N.'s PPA, there were no voxels showing a significant functional correlation to RSC, even with a liberal threshold ( $r=0.30, p<$ 0.01 , uncorrected). When C1's RSC was seeded, as expected, there were many voxels near C1's PPA showing reliable correlation to RSC responses. Similar results were found when PPA was selected as the seed; there were no voxels near J.N.'s RSC showing correlation to PPA. In C1, there were many voxels near C1's RSC showing correlation to PPA.

Also using the resting-state data, we examined the overall connectivity value for each seed region to determine the extent of the connectivity between this seed ROI (e.g., V1) and the other 12 target ROIs (average of V1's correlation to V2, V3, V4, V3a, V3b, MT, LOC, FFA, PPA, TOS, RSC, and HIP). This overall connectivity value for each seed ROI was compared between J.N. and controls (Fig. 8). Results showed that J.N.'s FFA, PPA, RSC, and HIP are significantly less correlated with the rest of the ROIs compared with controls' FFA, PPA, RSC, and HIP, respectively [FFA: J.N., $r=0.27$, controls, $r=0.68$ ( $\mathrm{SD}=0.09), p=0.001$; PPA: J.N., $r=0.35$, controls, $r=0.69$ (SD $=0.06), p<0.001$; RSC: J.N., $r=0.38$, controls, $r=0.54(\mathrm{SD}=0.07), p=0.04$, HIP: J.N., $r=0.20$, controls, $r=0.47(\mathrm{SD}=0.07), p=0.002]$. None of the other ROIs, including V1 $(p=0.63), \mathrm{V} 2(p=0.52), \mathrm{V} 3(p=$ $0.58), \mathrm{V} 4(p=0.64), \mathrm{V} 3 \mathrm{a}(p=0.58), \mathrm{V} 3 \mathrm{~b}(p=0.57), \mathrm{MT}(p=$ $0.52)$, LOC $(p=0.51)$, and TOS $(p=0.43)$, showed a reliable difference between J.N. and controls. Whereas J.N.'s early visual areas showed an overall correlation score that was comparable to that of controls, J.N.'s higher visual areas, including FFA, PPA, RSC, and HIP, showed a reliably reduced overall correlation value compared with that of the controls. This pattern of results can also be visualized in the height differences of J.N. and controls' ROIs in the hierarchical clustering results illustrated in Figure $5 C$. Whereas the heights are relatively similar and short for early visual areas, J.N.'s RSC, FFA, PPA, and HIP are taller than those of controls.

The functional connectivity results strongly suggest that, unlike controls, J.N.'s RSC and PPA are not functionally coupled. Together with the adaptation experiments, these fMRI results strongly point to J.N.'s RSC as being functionally anomalous relative to the controls' RSC profile.

\section{Structural connectivity}

Given the pronounced reduction in the functional connectivity between RSC and PPA, we investigated whether this disconnection might arise from a compromise in the structural integrity of the underlying WM tracts. Using a probabilistic tracking approach (Behrens et al., 2003, 2007), which computes a probabilistic estimate of the existence of a connection through two distant ROIs, we looked for those voxels showing reliable connectivity between RSC and PPA. All subjects showed highly similar tractography results. For controls, the path outputs were overlaid on top of each other and Figure $9 A$ shows those voxels that survived at least $54 \%$ overlap ( 7 of 13 ) across subjects. Figure $9 B$ shows the overlap between this output from controls and the result from J.N. The RSC and PPA paths are highly similar for J.N. and controls. The fractional anisotropy (FA) and mean diffusivity (MD) values from this RSC-PPA path were also highly similar across J.N. and controls $\left(t_{(12)}=\right.$ $-0.55, p=0.60$ for FA and $t_{(12)}=-0.60, p=66$ for MD, Figure $\left.9 C, D\right)$. To examine the overall structural connectivity pattern of RSC, the proportion connectivity of RSC voxels passing through the 12 target ROIs (V1-V4, V3a, V3b, MT, LOC, FFA, PPA, TOS, and HIP) was computed 
A JN's rPPA seeded
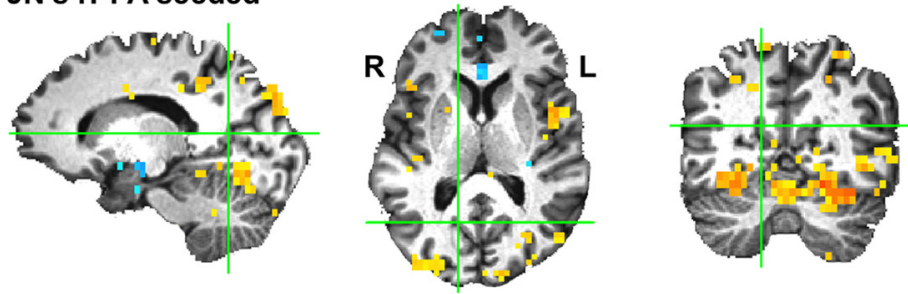

C1's rPPA seeded

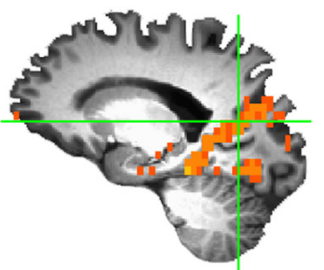

B JN's rRSC seeded
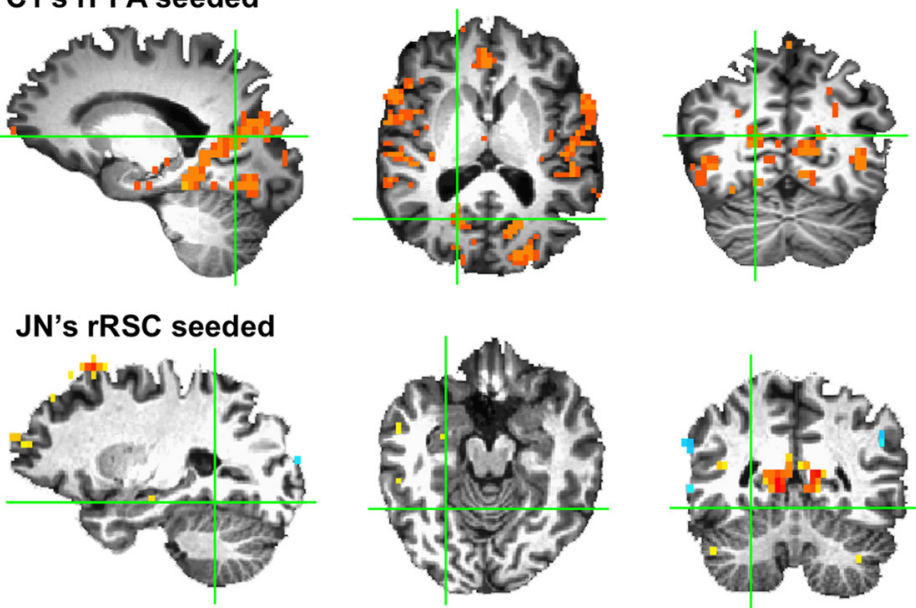

1.0

$0.0 \stackrel{\frac{\text { 음 }}{\frac{10}{2}}}{\frac{\text { 음 }}{3}}$

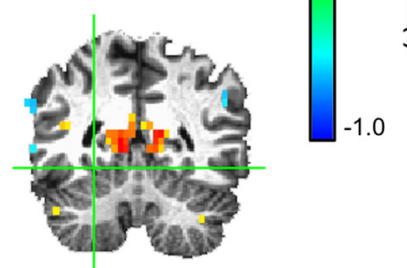

C1's rRSC seeded
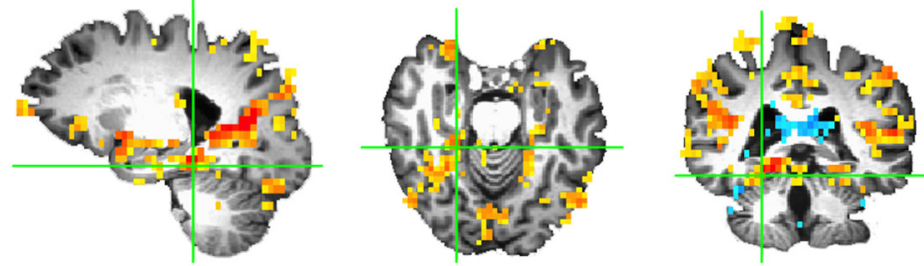

Figure 7. Visualization of whole-brain connectivity with PPA and RSC as seeds. $\boldsymbol{A}$, Whole-brain connectivity results in J.N. and $\mathrm{C} 1$ when the right PPA is seeded. The green crosshairs denote the center of each subject's respective right $\mathrm{RSC}$. Unlike C1, there were no voxels near RSC showing correlation to PPA responses in J.N. B, Similarly, the correlation results in J.N. and C1 are shown when the right RSC is seeded. The green crosshairs denote the centers of J.N. and C1's respective right PPA. Again, unlike (1, there were no voxels near J.N.'s PPA showing correlation to RSC responses. al., 2002; $r=0.57, p=0.03$ when excluding J.N. and $r=0.59, p=0.02$ when including J.N.). That is, across subjects, those individuals with higher functional and structural connectivity correlations were also those with higher navigability scores (Fig. 9H).

Unlike the functional connectivity results, the structural connectivity between J.N.'s RSC and PPA is highly similar to that of controls. This suggests that the atypical functional properties observed in J.N.'s RSC cannot be attributable to differences in the structural connectivity profile of RSC per se and are likely attributable to a functional alteration.

\section{Representative control C1}

As a visual benchmark to reference J.N.'s results, the results of $\mathrm{C} 1$ (who is age, handedness, and scanner matched to J.N.) are displayed in the figures as well. To ensure that $\mathrm{C} 1$ is a representative control, we compared C1's data with the rest of the controls' $(n=12)$ data. None of the following tests resulted in a significant difference: sizes of scene-selective ROIs $(0.26<$ $p<0.88)$, size of HIP $(p=0.65)$, gray matter volume of scene ROIs $(0.17<p<$ $0.54)$, HIP gray matter volume $(p=0.27)$, degree of adaptation effects in scene ROIs $(0.78<p<0.99$ for familiar and unfamiliar scenes and $0.73<p<0.90$ for indoor and outdoor scenes), functional connectivity rank scores between RSC and PPA using resting-state data ( $p=0.68$ ), functional connectivity rank scores between PPA and TOS $(p=0.61)$, and RSC structural connectivity patterns $(0.27<p<$ $0.99)$.
(Croxson et al., 2005). For each voxel in RSC, the number of total samples reaching a particular target (e.g., PPA) was compared with the total number of samples reaching any of the 12 target ROIs. J.N. and controls showed highly comparable results (Fig. 9E). The target ROI resulting in the highest proportion connectivity from RSC was HIP followed by PPA. The target ROIs with the lowest proportion connectivity from RSC were FFA and V4. None of the target ROIs differed reliably across J.N. and controls with respect to their proportion connectivity to RSC (V1: $t_{(12)}=-0.25, p=0.81 ; \mathrm{V} 2: t_{(12)}=-0.58, p=0.57 ; \mathrm{V} 3: t_{(12)}=-0.44$, $p=0.67$; V $: t_{(12)}=-0.91, p=0.65$; V3a: $t_{(12)}=0.43, p=0.67$; V3b: $t_{(12)}=-0.56, p=0.58$; MT: $t_{(12)}=0.92, p=0.38$; LOC: $t_{(12)}=1.11$, $p=0.29$; FFA: $t_{(12)}=0.41, p=0.69$; PPA: $t_{(12)}=0.20, p=0.85$; TOS: $t_{(12)}=0.17, p=0.87$; and HIP: $t_{(12)}=-0.14, p=0.89$ ).

In controls, there was a positive and significant correlation between the functional and structural connectivity of RSC to the remaining 12 target ROIs $(r=0.59, p=0.04$; Fig. $9 F)$. For example, if a particular target ROI (e.g., PPA) had high functional connectivity with RSC, then that target ROI also had high structural connectivity to RSC. This relationship was not present in J.N. $(r=-0.08, p=0.81$; Fig. $9 G)$. Importantly, the functional and structural connectivity relationship was positively correlated with behavioral navigability as assessed by the SBSDS (Hegarty et

\section{Comparison between age groups}

To ensure that control participants' data did not differ across the age-matched and non-age-matched groups, we compared younger participants' data (age range: $24-35$ years, mean $=29.0$, $n=7$ ) with the older participants' data (age range: $52-60$ years, mean $=56.3, n=6)$. None of the following tests resulted in a reliable difference: sizes of scene ROIs $(0.58<p<0.98)$, size of HIP $(p=0.18)$, gray matter volume of scene ROIs $(0.37<p<$ $0.83)$, HIP gray matter volume $(p=0.16)$, degree of adaptation effects in scene ROIs $(0.35<p<0.71$ for familiar and unfamiliar scenes and $0.50<p<0.66$ for indoor and outdoor scenes), functional connectivity rank scores between RSC and PPA using resting-state data $(p=0.17)$, functional connectivity rank scores between PPA and TOS ( $p=0.12)$, and RSC structural connectivity patterns $(0.07<p<0.96)$.

\section{Discussion}

The present study provides a comprehensive examination of the neural correlates of DTD, a disorder that markedly affects the ability of an individual to navigate successfully in the environment. The investigations conducted not only identify a potential neural correlate of DTD, but also provide insight into the neural 
functions that mediate normal navigation. Although the response properties of the scene-selective areas PPA, TOS, and RSC have been widely studied in healthy controls (Epstein and Kanwisher, 1998; Hasson et al., 2003), no study has investigated scene-selective ROIs in DTDs either in terms of their anatomical or structural characteristics or their functional properties. Uncovering the neural correlate of DTD is useful in two main respects. First, this provides a thorough description of the neural perturbations that accompany the sometimes-debilitating impairment in navigation experienced by these individuals. Because J.N. is a representative case of DTD, the current study has the opportunity to improve our understanding of this atypical profile on both a behavioral and neural level. Second, from a basic science perspective, identifying deviations in the neural profile in DTD affords a deeper insight into the computational contribution of particular region(s) under investigation and a potential explanation for its causal role in DTD. Our study reports the results of detailed behavioral and neural examination conducted with J.N., a representative single patient with DTD. The major and novel result was that there was an atypical response profile of the RSC and there was atypical functional coupling between RSC and PPA. These findings indicate that RSC is a particularly vulnerable component of the scene and navigation network in DTD.

Using a functional localizer, we identified PPA, TOS, and RSC bilaterally in J.N. and all were comparable to the corresponding regions in healthy controls in terms of size, cortical thickness, and degree of scene selectivity. The key results emerged when examining the adaptation profile of scene-selective regions, specifically the RSC. Although J.N.'s PPA, TOS, and RSC did not differ in the overall responsiveness to scenes, relative to controls, unlike the PPA and TOS, the RSC did not exhibit repetition attenuation to repeated scenes. This finding was consistent across two adaptation experiments and points to the abnormal functional profile of J.N.'s RSC. The functional connectivity results from the restingstate data, as well as from the adaptation experiments, provide consistent evidence for the strikingly reduced functional coupling between J.N.'s RSC and PPA. Unlike J.N., every control participant demonstrated high functional connectivity between RSC and PPA and this was highly reliable across independent datasets.

The reduced functional coupling between J.N.'s RSC and PPA cannot be explained by differences in the structural connectivity between these two regions, as evidenced by the largely overlapping RSC-PPA tractography between J.N. and controls. In addition, J.N.'s structural connectivity profile of RSC to the remaining ROIs was highly consistent with that of the controls. These results critically point to the functional deficits in RSC as being the likely neural correlate subserving the navigational deficits. In control subjects, RSC's structural and functional connectivity profiles were highly correlated and the degree of the structural and functional coupling was predictive of navigability.

The behavioral navigation deficits shown in J.N. are most similar to the navigational deficits witnessed in patients with explicit lesions to the RSC (Takahashi et al., 1997; Maguire, 2001). Like these patients, J.N. is able to recognize familiar buildings and landmarks and she does not have major deficits in visual form recognition or in general memory. J.N. is, however, severely impaired in using spatial information even in familiar environments (e.g., she constantly gets lost in the neighborhood she has lived in for years) and performs poorly on tasks requiring the mental manipulation of spatial information (e.g., the card rotation, paper-folding, and mental rotation tests) and spatial updating (e.g., card placement task and deficits in tests of forming a cognitive map). The fMRI results demonstrating a functional deficit in J.N.'s RSC are also consistent with the hypothesis that the neural correlates for topographic disorientation stem from a functionally deviant RSC. Although J.N. performed within the normal range in the scene classification and landmark recognition tests, a more fine-grained testing of scene recognition and analysis may be required to sufficiently argue for J.N.'s intact scene recognition. At present, our testing provides a broad stroke analysis of J.N.'s scene perception.

The atypical neural profile in J.N. appears largely restricted to the RSC. Iaria and colleagues (2009), however, found decreased activity in the DTD's left HIP in addition to that of RSC bilaterally while participants performed a spatial navigation task. However, because there were clear behavioral differences in the DTD's and the controls' ability to perform the navigation task, it is unclear whether the neural differences observed were solely attributable to the navigation deficits in the DTD or were a result of confounding factors such as differences in task difficulty, different behavioral strategies, or compensatory mechanisms (Palermo et al., 2014). The fMRI experiments in the current investigation did not require participants to explicitly engage in a navigation or a spatial manipulation task (specifically to avoid the confound of different behavioral competence). Our results offer complementary and converging evidence from the adaptation and restingstate experiments that J.N.'s RSC is functionally deviant. We have also gone beyond characterizing the BOLD signal in J.N. as was done in the previous study by examining functional and structural connectivity in tandem.

The RSC atypicality that was observed is evident bilaterally in J.N. and this contrasts with the fact that the lesion in cases with acquired topographic disorientation due to retrosplenial damage is usually unilateral and to the right hemisphere. Interestingly, in many of these latter cases, the navigation deficits generally resolve over time (Maguire, 2001), suggesting that the left RSC or other nodes in the right hemisphere may compensate for the retro- 


\section{A controls' RSC and PPA paths}

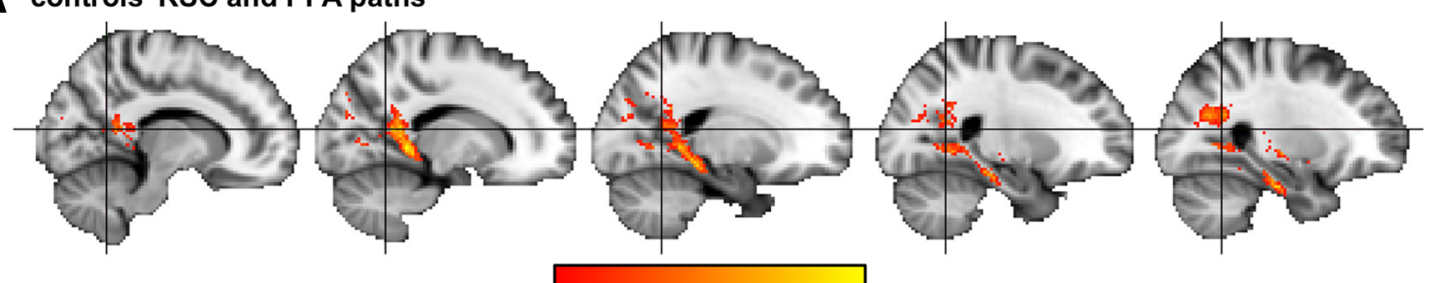

$54 \%$ subjects overlap $100 \%$

B JN's RSC and PPA paths
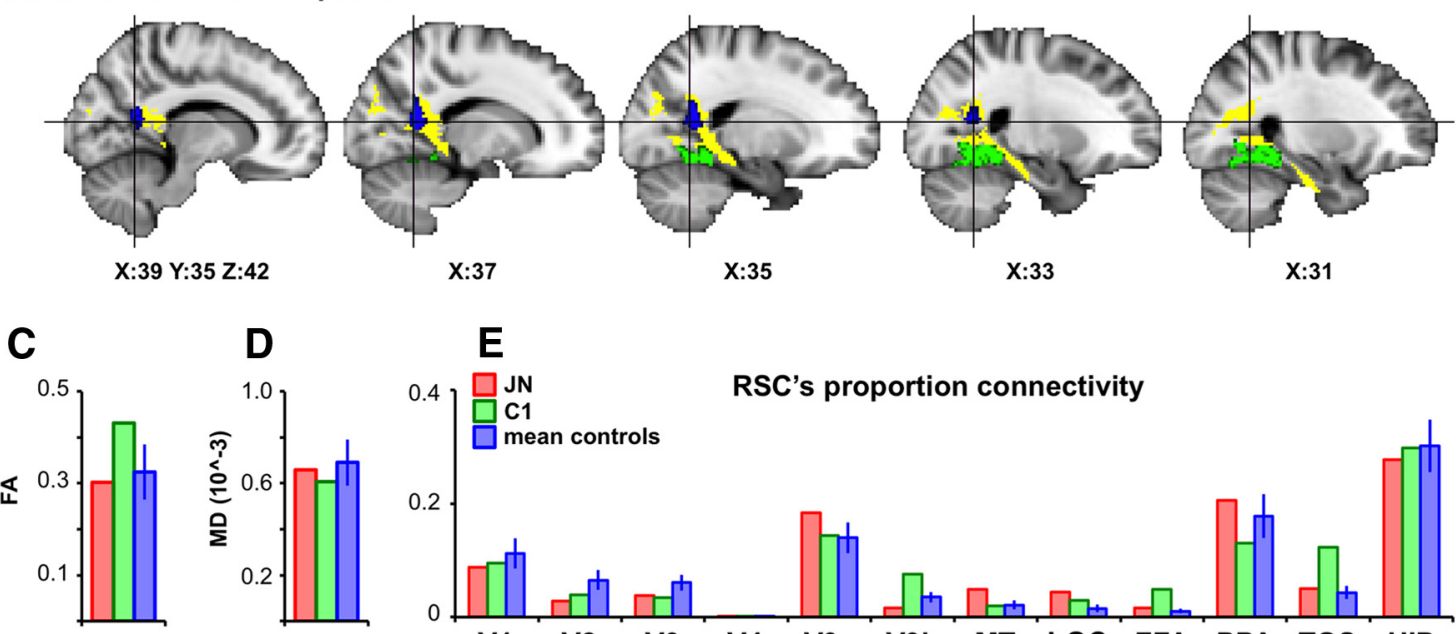

E

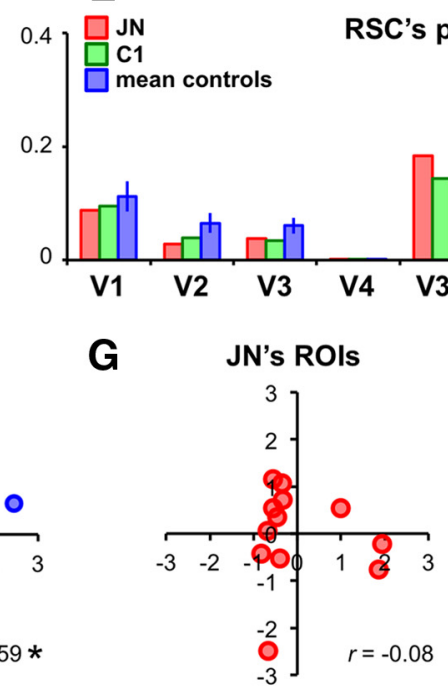

RSC's proportion connectivity

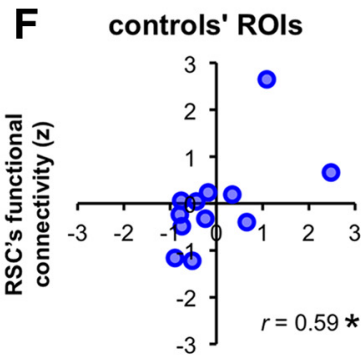

RSC's structural connectivity (z)

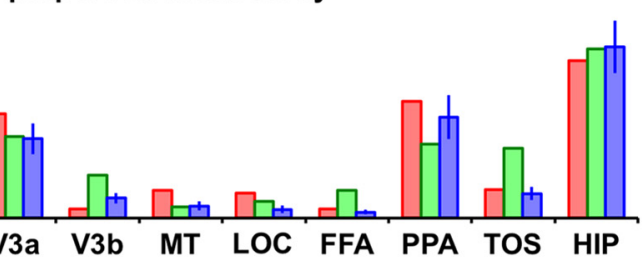

\section{H}

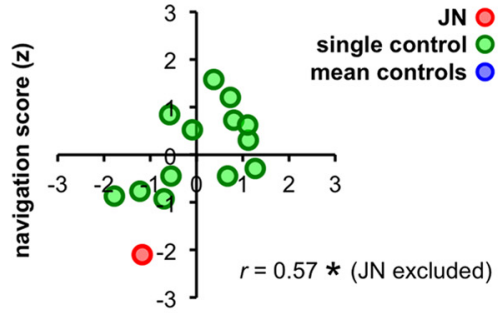

RSC's structural-functional coupling (z)

Figure 9. Structural connectivity, functional connectivity, and navigability. A, WM tracts connecting RSC and PPA that were consistent across at least $50 \%$ of the controls displayed in MNI space. $B$, Yellow voxels denote the overlap between J.N.'s RSC-PPA path result and controls' from A. J.N.'s RSC is shown in blue and PPA in green. C, D, FA and MD values extracted from the RSC-PPA tracks among J.N., $C 1$, and averaged controls. $\boldsymbol{E}$, Pattern of the structural connectivity between RSC and the rest of the ROls were highly similar across J.N. and controls. $\boldsymbol{F}$, Controls' structural and functional connectivity patterns of RSC were positively correlated. Blue dots denote individual target ROI's (averaged across controls) connectivity to RSC. G, J.N.'s functional and structural connectivity patterns of RSC were not correlated. $\boldsymbol{H}$, Structural and functional coupling of RSC's connectivity to other ROls correlated positively with navigation ability, as assessed by SBSDS. Values plotted in $\boldsymbol{F}-\boldsymbol{H}$ are $z$-scores.

splenial functions. DTD individuals, on the other hand, suffer from a life-long deficit, potentially as a result of the bilateral nature of the RSC functional deficits and the inability to compensate for the deficit. It is also possible that, unlike acquired topographic disorientation, DTDs suffer from a life-long deficit due to the impairments in the neural processes that allow compensation after RSC dysfunctions.

What might be the contribution of the RSC (and its subsequent dysfunction in DTD)? The ability to navigate relies on a flexible mechanism of representing multiple complementary reference frames. View-specific egocentric representations allow for updating self-motion and allocentric representations allow for understanding the relative positions between landmarks that are accessible across varying viewpoints (e.g., the bank is east of the park). One functional role of RSC proposed in spatial cognition (Burgess, 2006, 2008) is in the transformation of egocentric rep- resentations mediated by the parietal cortex (Ino et al., 2002; Ciaramelli et al., 2010) to allocentric representations mediated by medial temporal structures such as the HIP (Rolls et al., 1997; Ekstrom et al., 2003) and vice versa. RSC evinces sensitivity to both egocentric and allocentric representations and may well support the conversion of visual stimuli into different reference frames. Indeed, anatomical connectivity studies from macaques demonstrate that RSC has strong connectivity to both parietal and medial temporal regions (Lavenex et al., 2004; Kobayashi and Amaral, 2000; Kravitz et al., 2011) and this puts RSC in the prime location for the functional role of translating between different coordinate frames. Therefore, the DTD's RSC might reflect the incorrect or inefficient computations involved in the transformation of reference frames, which would prohibit flexible and efficient spatial representations. Previous studies (Iaria and Barton, 2010) have carefully characterized the behavioral deficits in a 
number of cognitive processes related to navigation: heading orientation, left/right orientation, landmark recognition, and path formation. All of these processes are related to the DTD individuals' difficulty in forming a cognitive map, a skill that requires converting visual input of the local environment into an allocentric spatial representation - which is consistent with an atypical functioning RSC. Indeed, J.N. and DTDs were impaired in forming and using a cognitive map, as evidenced using the online perceptual battery (Iaria and Barton, 2010). J.N. was also impaired in the card placement task that required the use of an allocentric spatial representation, a task that has also been shown to be impaired in those with RSC damage (Hashimoto et al., 2010). In addition, the ability to transform a spatial map from one viewpoint to another was impaired in J.N., as shown in the card rotation task, paper-folding task, and mental rotation. Therefore, this study has demonstrated, not only the neural atypicality of RSC, but also a behavioral profile that reflects the outcome of this neural alteration.

In summary, the current study offers converging evidence that the scene and navigation network, especially the RSC, is functionally deviant in a DTD individual and implicates an alteration in bilateral RSC as a potential neural basis for navigational deficits in DTDs. These results provide direct leads for important future studies to address the precise relationship between the representations in RSC and navigation. For example, further research is needed to understand the relationship between functional coupling and the adaptation effects in the scene ROIs. A further topic of interest concerns the directionality of the RSC and PPA relationship: although we report consistently reduced functional connectivity between J.N.'s RSC and PPA, it is possible that the lack of the adaptation effects in RSC is a consequence of the reduced inputs from PPA or of the reduced functional coupling between RSC and PPA. Determining causality will elucidate further the neural system subserving navigation. Last, future investigations can examine the representation of spatial maps within multivoxel patterns of activity within the RSC in DTDs, which recently has provided insight into the function of the RSC is normal navigators (Marchette et al., 2014). The present investigation provides the most extensive neuroimaging study on the neural mechanisms underlying DTD completed to date and sheds light on the neural substrates mediating intact navigation.

\section{References}

Aguirre GK, D’Esposito M (1999) Topographical disorientation: a synthesis and taxonomy. Brain 122:1613-1628. CrossRef Medline

Aguirre GK, Zarahn E, D’Esposito M (1998) An area within human ventral cortex sensitive to "building" stimuli: evidence and implications. Neuron 21:373-383. CrossRef Medline

Al-Aidroos N, Said CP, Turk-Browne NB (2012) Top-down attention switches coupling between low-level and high-level areas of human visual cortex. Proc Natl Acad Sci U S A 109:14675-14680. CrossRef Medline

Arcaro MJ, McMains SA, Singer BD, Kastner S (2009) Retinotopic organization of human ventral visual cortex. J Neurosci 29:10638-10652. CrossRef Medline

Avidan G, Hasson U, Malach R, Behrmann M (2005) Detailed exploration of face-related processing in congenital prosopagnosia: 2. Functional neuroimaging findings. J Cogn Neurosci 17:1150-1167. CrossRef Medline

Bandettini PA, Jesmanowicz A, Wong EC, Hyde JS (1993) Processing strategies for time-course data sets in functional MRI of the human brain. Magn Reson Med 30:161-173. CrossRef Medline

Behrens TE, Woolrich MW, Jenkinson M, Johansen-Berg H, Nunes RG, Clare S, Matthews PM, Brady JM, Smith SM (2003) Characterization and propagation of uncertainty in diffusion-weighted MR Imaging. Magn Reson Med 50:1077-1088. CrossRef Medline

Behrens TE, Berg HJ, Jbabdi S, Rushworth MF, Woolrich MW (2007) Prob- abilistic diffusion tractography with multiple fibre orientations: what can we gain? Neuroimage 34:144-155. CrossRef Medline

Behrmann M, Kimchi R (2003) What does visual agnosia tell us about perceptual organization and its relationship to object perception? J Exp Psychol Hum Percept Perform 29:19-42. CrossRef Medline

Behrmann M, Peterson MA, Moscovitch M, Suzuki S (2006) Independent representation of parts and the relations between them: Evidence from integrative agnosia. J Exp Psychol Hum Percept Perform 32:1169-1184. CrossRef Medline

Bianchini F, Incoccia C, Palermo L, Piccardi L, Zompanti L, Sabatini U, Peran P, Guariglia C (2010) Developmental topographical disorientation in a healthy subject. Neuropsychologia 48:1563-1573. CrossRef Medline

Bianchini F, Palermo L, Piccardi L, Incoccia C, Nemmi F, Sabatini U, Guariglia C (2014) Where am I? A new case of developmental topographical disorientation. J Neuropsychol 8:107-124. CrossRef Medline

Burgess N (2006) Spatial memory: how egocentric and allocentric combine. Trends Cogn Sci 10:551-557. CrossRef Medline

Burgess N (2008) Spatial cognition and the brain. Ann N Y Acad Sci 1124: 77-97. CrossRef Medline

Cabeza R, Prince SE, Daselaar SM, Greenberg DL, Budde M, Dolcos F, LaBar KS, Rubin DC (2004) Brain activity during episodic retrieval of autobiographical and laboratory events: an fMRI study using a novel photo paradigm. J Cogn Neurosci 16:1583-1594. CrossRef Medline

Ciaramelli E, Rosenbaum RS, Solcz S, Levine B, Moscovitch M (2010) Mental space travel: Damage to posterior parietal cortex prevents egocentric navigation and reexperiencing of remote spatial memories. J Exp Psychol Learn Mem Cogn 36:619-634. CrossRef Medline

Crawford JR, Howell DC (1998) Regression equations in clinical neuropsychology: an evaluation of statistical methods for comparing predicted and obtained scores. J Clin Exp Neuropsychol 20:755-762. CrossRef Medline

Crawford JR, Garthwaite PH, Howell DC, Gray CD (2004) Inferential methods for comparing a single case with a control sample: modified t-tests versus Mycroft et al.'s (2002) modified ANOVA. Cogn Neuropsychol 21:750-755. CrossRef Medline

Croxson PL, Johansen-Berg H, Behrens TE, Robson MD, Pinsk MA, Gross CG, Richter W, Richter MC, Kastner S, Rushworth MF (2005) Quantitative investigation of connections of the prefrontal cortex in the human and macaque using probabilistic diffusion tractography. J Neurosci 25 : 8854-8866. CrossRef Medline

Dilks DD, Julian JB, Paunov AM, Kanwisher N (2013) The occipital place area is causally and selectively involved in scene perception. J Neurosci 33:1331-1336a. CrossRef Medline

Duchaine B, Nakayama K (2006) The Cambridge Face Memory Test: Results for neurologically intact individuals and an investigation of its validity using inverted face stimuli and prosopagnosic participants. Neuropsychologia 44:576-585. CrossRef Medline

Ekstrom AD, Kahana MJ, Caplan JB, Fields TA, Isham EA, Newman EL, Fried I (2003) Cellular networks underlying human spatial navigation. Nature 425:184-188. CrossRef Medline

Ekstrom RB, French JW, Harman H, Dermen D (1976) Manual for the kit of factor-referenced cognitive tests. Princeton, NJ: Educational Testing Service.

Epstein RA (2008) Parahippocampal and retrosplenial contributions to human spatial navigation. Trends Cogn Sci 12:388-396. CrossRef Medline

Epstein RA, Morgan LK (2012) Neural responses to visual scenes reveals inconsistencies between fMRI adaptation and multivoxel pattern analysis. Neuropsychologia 50:530-543. CrossRef Medline

Epstein R, Kanwisher N (1998) A cortical representation of the local visual environment. Nature 392:598-601. CrossRef Medline

Epstein RA, Higgins JS, Jablonski K, Feiler AM (2007a) Visual scene processing in familiar and unfamiliar environments. J Neurophysiol 97: 3670-3683. CrossRef Medline

Epstein RA, Parker WE, Feiler AM (2007b) Where am I now? Distinct roles for parahippocampal and retrosplenial cortices in place recognition. J Neurosci 27:6141-6149. CrossRef Medline

Epstein RA, Parker WE, Feiler AM (2008) Two kinds of FMRI repetition suppression? Evidence for dissociable neural mechanisms. J Neurophysiol 99:2877-2886. CrossRef Medline

Epstein R, Deyoe EA, Press DZ, Rosen AC, Kanwisher N (2001) Neuropsychological evidence for a topographical learning mechanism in parahippocampal cortex. Cogn Neuropsychol 18:481-508. CrossRef Medline

Epstein R, Graham KS, Downing PE (2003) Viewpoint-specific scene rep- 
resentations in human parahippocampal cortex. Neuron 37:865-876. CrossRef Medline

Fischl B, Salat DH, Busa E, Albert M, Dieterich M, Haselgrove C, van der Kouwe A, Killiany R, Kennedy D, Klaveness S, Montillo A, Makris N, Rosen B, Dale AM (2002) Whole brain segmentation: automated labeling of neuroanatomical structures in the human brain. Neuron 33:341355. CrossRef Medline

Habib M, Sirigu A (1987) Pure topographical disorientation: a definition and anatomical basis. Cortex 23:73-85. CrossRef Medline

Hashimoto R, Tanaka Y, Nakano I (2010) Heading disorientation: A new test and a possible underlying mechanism. Eur Neurol 63:87-93. CrossRef Medline

Hasson U, Harel M, Levy I, Malach R (2003) Large-scale mirror-symmetry organization of human occipito-temporal object areas. Neuron 37:10271041. CrossRef Medline

Hegarty M, Richardson AE, Montello DR, Lovelace K, Subbiah I (2002) Development of a self-report measure of environmental spatial ability. Intelligence 30:425-447. CrossRef

Iaria G, Barton JJ (2010) Developmental topographical disorientation: a newly discovered cognitive disorder. Exp Brain Res 206:189-196. CrossRef Medline

Iaria G, Bogod N, Fox CJ, Barton JJ (2009) Developmental topographical disorientation: case one. Neuropsychologia 47:30-40. CrossRef Medline

Iaria G, Arnold AE, Burles F, Liu I, Slone E, Barclay S, Bech-Hansen TN, Levy RM (2014) Developmental topographic disorientation and decreased hippocampal functional connectivbity. Hippocampus 24:1364-1374. CrossRef Medline

Ino T, Inoue Y, Kage M, Hirose S, Kimura T, Fukuyama H (2002) Mental navigation in humans is processed in the anterior bank of the parietooccipital sulcus. Neurosci Lett 322:182-186. Medline

Johnson SC (1967) Hierarchical clustering schemes. Psychometrika 32: 241-254. CrossRef Medline

Katayama K, Takahashi N, Ogawara K, Hattori T (1999) Pure topographical disorientation due to right posterior cingulate lesion. Cortex 35:279-282. CrossRef Medline

Kim JG, Biederman I (2011) Where do objects become scenes? Cereb Cortex 21:1738-1746. CrossRef Medline

Kim JG, Biederman I, Lescroart MD, Hayworth KJ (2009) Adaptation to objects in the lateral occipital complex (LOC): Shape or semantics? Vision Res 49:2297-2305. CrossRef Medline

Kobayashi Y, Amaral DG (2000) Macaque monkey retrosplenial cortex: I. Three-dimensional and cytoarchitectonic organization. J Comp Neurol 426:339-365. CrossRef Medline

Konen CS, Behrmann M, Nishimura M, Kastner S (2011) The functional neuroanatomy of object agnosia: a case study. Neuron 71:49-60. CrossRef Medline

Kozhevnikov M, Hegarty M (2001) A dissociation between object manipulation spatial ability and spatial orientation ability. Mem Cognit 29:745756. CrossRef Medline

Kravitz DJ, Saleem KS, Baker CI, Mishkin M (2011) A new neural framework for visuospatial processing. Nat Rev Neurosci 12:217-230. CrossRef Medline

Lavenex P, Suzuki WA, Amaral DG (2004) Perirhinal and parahippocampal cortices of the macaque monkey: intrinsic projections and interconnections. J Comp Neurol 472:371-394. Medline
Maguire EA (2001) The retrosplenial contribution to human navigation: a review of lesion and neuroimaging findings. Scand J Psychol 42:225-238. CrossRef Medline

Marchette SA, Vass LK, Ryan J, Epstein RA (2014) Anchoring the neural compass: coding of local spatial reference frames in human medial parietal lobe. Nat Neurosci 17:1598-1606. CrossRef Medline

Mendez MF, Cherrier MM (2003) Agnosia for scenes in topographagnosia. Neuropsychologia 41:1387-1395. CrossRef Medline

Norman-Haignere SV, McCarthy G, Chun MM, Turk-Browne NB (2012) Category-selective background connectivity in ventral visual cortex. Cereb Cortex 22:391-402. CrossRef Medline

Oldfield RC (1971) The assessment and analysis of handedness: the Edinburgh inventory. Neuropsychologia 9:97-113. CrossRef Medline

Palermo L, Foti F, Ferlazzo F, Guariglia C, Petrosini L (2014) I find my way in a maze but not in my own territory! Navigational processing in developmental topographical disorientation. Neuropsychology 28:135-146. CrossRef Medline

Pinsk MA, Arcaro M, Weiner KS, Kalkus JF, Inati SJ, Gross CG, Kastner S (2009) Neural representations of faces and body parts in macaque and human cortex: a comparative fMRI study. J Neurophysiol 101:2581-2600. Medline

Podzebenko K, Egan GF, Watson JD (2002) Widespread dorsal stream activation during a parametric mental rotation task, revealed with functional magnetic resonance imaging. Neuroimage 15:547-558. CrossRef Medline

Rilling JK, Glasser MF, Preuss TM, Ma X, Zhao T, Hu X, Behrens TE (2008) The evolution of the arcuate fasciculus revealed with comparative DTI. Nat Neurosci 11:426-428. CrossRef Medline

Rolls ET, Robertson RG, Georges-François P (1997) Spatial view cells in the primate hippocampus. Eur J Neurosci 9:1789-1794. CrossRef Medline

Schneider KA, Richter MC, Kastner S (2004) Retinotopic organization and functional subdivisions of the human lateral geniculate nucleus: a highresolution functional magnetic resonance imaging study. J Neurosci 24: 8975-8985. CrossRef Medline

Sholl MJ, Kenny RJ, DellaPorta KA (2006) Allocentric-heading recall and its relation to self-reported sense-of-direction. J Exp Psychol Learn Mem Cogn 32:516-533. CrossRef Medline

Stark M (1996) Impairment of an egocentric map of locations: implications for perception and action. Cogn Neuropsychol 13:481-524. CrossRef

Swisher JD, Halko MA, Merabet LB, McMains SA, Somers DC (2007) Visual topography of human intraparietal sulcus. J Neurosci 27:5326-5337. CrossRef Medline

Takahashi N, Kawamura M, Shiota J, Kasahata N, Hirayama K (1997) Pure topographic disorientation due to right retrosplenial lesion. Neurology 49:464-469. CrossRef Medline

Ward EJ, Chun MM, Kuhl BA (2013) Repetition suppression and multivoxel pattern similarity differentially track implicit and explicit visual memory. J Neurosci 33:14749-14757. CrossRef Medline

Wechsler D (1997) WMS-III: Wechsler memory scale administration and scoring manual, 3rd ed. San Antonio, TX: Psychological Corporation.

Whiteley AM, Warrington EK (1978) Selective impairment of topographical memory: a single case study. J Neurol Neurosurg Psychiatry 41:575578. CrossRef Medline 